\title{
Neural Activity in the Ventral Pallidum Encodes Variation in the Incentive Value of a Reward Cue
}

\author{
Allison M. Ahrens, ${ }^{1}$ Paul J. Meyer, ${ }^{1,2}$ Lindsay M. Ferguson, ${ }^{1}$ Terry E. Robinson, ${ }^{1}$ and J. Wayne Aldridge ${ }^{1}$ \\ ${ }^{1}$ Department of Psychology, University of Michigan, Ann Arbor, Michigan 48109, and ${ }^{2}$ Department of Psychology, University at Buffalo, Buffalo, New York \\ 14051
}

There is considerable individual variation in the extent to which reward cues are attributed with incentive salience. For example, a food-predictive conditioned stimulus (CS; an illuminated lever) becomes attractive, eliciting approach toward it only in some rats ("sign trackers," STs), whereas others ("goal trackers," GTs) approach the food cup during the CS period. The purpose of this study was to determine how individual differences in Pavlovian approach responses are represented in neural firing patterns in the major output structure of the mesolimbic system, the ventral pallidum (VP). Single-unit in vivo electrophysiology was used to record neural activity in the caudal VP during the performance of ST and GT conditioned responses. All rats showed neural responses to both cue onset and reward delivery but, during the CS period, STs showed greater neural activity than GTs both in terms of the percentage of responsive neurons and the magnitude of the change in neural activity. Furthermore, neural activity was positively correlated with the degree of attraction to the cue. Given that the CS had equal predictive value in STs and GTs, we conclude that neural activity in the VP largely reflects the degree to which the CS was attributed with incentive salience.

Key words: goal tracking; motivation; Pavlovian conditioning; rats; sign tracking; ventral pallidum

\section{Significance Statement}

Cues associated with reward can acquire motivational properties (i.e., incentive salience) that cause them to have a powerful influence on desire and motivated behavior. There are individual differences in sensitivity to reward-paired cues, with some individuals attaching greater motivational value to cues than others. Here, we investigated the neural activity associated with these individual differences in incentive salience. We found that cue-evoked neural firing in the ventral pallidum (VP) reflected the strength of incentive motivation, with the greatest neural responses occurring in individuals that demonstrated the strongest attraction to the cue. This suggests that the VP plays an important role in the process by which cues gain control over motivation and behavior.

\section{Introduction}

If cues associated with rewards are attributed with incentive salience, they come to exert powerful control over motivated behavior (Cardinal et al., 2002; Robinson and Berridge, 2008). Some individuals experience cue-induced incentive motivation more strongly than others and are more susceptible to the "irre-

Received March 5, 2016; revised May 20, 2016; accepted June 9, 2016.

Author contributions: A.M.A., P.J.M., T.E.R., and J.W.A. designed research; A.M.A. and L.M.F. performed research; A.M.A. and L.M.F. analyzed data; A.M.A., P.J.M., T.E.R., and J.W.A. wrote the paper.

This work was supported by the National Institute on Drug Abuse (Grant P01 DA031656 to T.E.R./J.W.A. and Grants T32 DA007267 and T32 DA007268 to A.M.A.). The content is solely the responsibility of the authors and does not necessarily represent the official views of the National Institute on Drug Abuse or the National Institutes of Health. We thank Steve Zekany, Marc Bradshaw, Lauren Longyear, Shani Ross, and Christy Itoga for assistance on this project.

The authors declare no competing financial interests.

Correspondence should be addressed to Dr. Allison M. Ahrens, Department of Psychology, University of Michigan, 530 Church St., Ann Arbor Ml, 48109. E-mail: ahrensam@umich.edu.

DOI:10.1523/JNEUROSCI.0736-16.2016

Copyright $\odot 2016$ the authors $\quad 0270-6474 / 16 / 367957-14 \$ 15.00 / 0$ sistible pull" of food- or drug-associated cues (Robinson and Flagel, 2009; Saunders and Robinson, 2013; Robinson et al., 2014). For example, in rats, if a discrete cue (a lever conditioned stimulus, CS) predicts food reward (unconditioned stimulus, US), some rats come to approach and engage the lever CS (signtrackers, STs), others approach the site of food delivery (goaltrackers, GTs) during the CS period, and yet others vacillate (intermediates, INs). Therefore, the lever is a predictive CS in both STs and GTs, eliciting a conditioned response (CR) in both, but it is a more attractive incentive stimulus in STs than in GTs (Tomie et al., 2000, 2008; Flagel et al., 2009; Saunders and Robinson, 2010, 2013; Meyer et al., 2012, 2014; Saunders et al., 2013; Robinson et al., 2014).

Mesolimbic structures are critical for many aspects of learning and motivation, and cue-induced neural firing in mesolimbic structures has been measured using electrophysiology during a variety of appetitive tasks (Schultz et al., 1997; Waelti et al., 2001; Ambroggi et al., 2011; McGinty et al., 2013), including Pavlovian 
conditioned approach (PCA) (Day et al., 2006; Wan and Peoples, 2006). However, in the majority of studies, the predictive and incentive features of Pavlovian cues are confounded because they frequently co-occur. The ST/GT model provides a way to parse the predictive and incentive value of reward cues and thus the neurobiological substrate of each (Flagel et al., 2011b). For example, previous studies showed that dopamine is necessary for the acquisition and performance of ST, but not GT, CRs (Flagel et al., 2011a, 2011b; Saunders and Robinson, 2012; Danna et al., 2013; Haight et al., 2015; Yager et al., 2015) and that, with learning, a phasic dopamine signal transfers from the US to the CS in STs, but not GTs (Flagel et al., 2011b). Here, we sought to determine whether cue-evoked neural activity in the ventral pallidum (VP) also reflects variation in the extent to which the cue acquires motivational (attractive) properties.

The VP is the primary output structure of the mesolimbic system and is thought to be a major hub where motivational signals are translated into appetitive behavior (Smith et al., 2009). The VP is involved in several aspects of reward-related behavior, including hedonic facial reactions to palatable tastes (Cromwell and Berridge, 1993; Smith and Berridge, 2005, 2007; Shimura et al., 2006; Tindell et al., 2006; Ho and Berridge, 2013; Castro and Berridge, 2014), the reinstatement of cocaine-seeking behavior (Stefanik et al., 2013; Mahler et al., 2014), and Pavlovian-toinstrumental transfer effects (Leung and Balleine, 2013). In addition, disruption of the VP has been shown to reduce the acquisition of ST, but not GT, behavior (Chang et al., 2015). The current study focused only on the posterior region of the VP because a number of studies have found that reward-paired cues elicit robust changes in neural activity in this area (Tindell et al., 2005, 2006, 2009; Smith et al., 2011; Tachibana and Hikosaka, 2012; Avila and Lin, 2014a, 2014b) and, importantly, this is the only brain region that has been shown to encode the predictive and incentive value of cues in a dissociable way (Tindell et al., 2005, 2009; Zhang et al., 2009; Smith et al., 2011). We hypothesized, therefore, that the neural representation of incentive salience is encoded in the VP, so cue-induced VP firing would be represented differently in STs and GTs.

\section{Materials and Methods}

Animals

A total of 28 male rats (26 from Harlan Laboratories, two from Charles River Laboratories), weighing 250-275 g on arrival, were singly housed in standard polycarbonate cages with corncob bedding. Rats were housed under a reverse $12 \mathrm{~h}$ light/dark cycle, with all testing taking place during the dark phase of the cycle. Water and standard rat chow were available ad libitum throughout the experiment (i.e., rats were not food restricted at any time) and testing began after 1 week of acclimation and handling. All procedures were approved by the University of Michigan Committee on the Use and Care of Animals.

\section{Surgery}

Animals were anesthetized with isoflurane (3.5\% induction, $1.5-2 \%$ maintenance). Incisions were made at the top of the skull and the analgesic sensoricaine $(0.1 \mathrm{ml}$ of $0.25 \%)$ was administered into the incision to provide local pain relief. Electrode bundles were implanted bilaterally into the posterior $\mathrm{VP}(\mathrm{AP}=-0.7$ to $-1 \mathrm{~mm} ; \mathrm{ML}=2.5-3 \mathrm{~mm})$ using a stereotaxic apparatus. The tip of wire bundles were initially implanted 1 $\mathrm{mm}$ above the $\mathrm{VP}(\mathrm{DV}=6.6 \mathrm{~mm})$ and later advanced throughout the dorsal-ventral range of the VP target $(\mathrm{DV}=-7.6$ to $-8.6 \mathrm{~mm}$; see neural recording procedures). Microdrives were affixed to the skull with bone screws and acrylic cement, with a grounding wire soldered to one of the bone screws. For $2 \mathrm{~d}$ after surgery, rats received penicillin $(0.1 \mathrm{ml}$, s.c. $)$ to prevent infection and flunixin $(2.5 \mathrm{mg} / \mathrm{kg}$, i.p.) to provide pain relief. Testing resumed after 1 week of recovery.

\section{Apparatus}

All experiments took place in standard test chambers $(30.5 \times 24.1 \times 21$ $\mathrm{cm}$; Med Associates) with modified open tops located inside soundattenuating cabinets. Each chamber contained a pellet dispenser connected to a pellet magazine that extended $3 \mathrm{~cm}$ into the chamber (Med Associates). The pellet magazine was mounted in the center of the front wall and equipped with an infrared sensor to record magazine head entries. A single retractable lever with LED backlight (Coulbourn Instruments) was mounted to the left of the magazine. Chambers also contained a red house light mounted at the top of the back wall that was constantly illuminated during all sessions. A white noise generator (Med Associates) was mounted at the back of the chamber to mask background noise. Custom software (Mtask; The Aldridge Lab) controlled the trials and recorded lever presses and magazine entries.

\section{PCA}

Before the start of training, rats were given 25 banana pellets in their home cages for $2 \mathrm{~d}$ ( $45 \mathrm{mg}$ of banana-flavored pellets; BioServ, \#F0059). On a single pretraining day, 25 pellets were delivered on a variable time (VT-30 s) schedule without the lever CS to train rats to retrieve pellets from the magazine. PCA training began the next day. On PCA training days, rats were first placed in the chamber for a $1 \mathrm{~min}$ habituation period with lights off. The red house light and white noise turned on when the session began and remained on for the rest of the session. Each session had 25 trials separated by VT-90 (30-150 s) intervals. In each trial, the CS was the insertion of the lever (and the illumination of the LED behind the lever) into the chamber for $8 \mathrm{~s}$. After $8 \mathrm{~s}$, the lever retracted and a pellet was dispensed simultaneously. An additional unpaired control group underwent the same procedures except that, during PCA sessions, the 25 lever presentation and 25 pellets occurred on a pseudorandom schedule and never coincided. Lever presses were recorded during the $8 \mathrm{~s}$ CS period when the lever was present and magazine entries were recorded throughout the session.

\section{Video analysis}

PCA sessions were recorded with four cameras positioned around the chamber. Two cameras were mounted at the side of the chamber with a close-up view of both the lever and the food cup and one was mounted at the top of the chamber with a view of the entire chamber. For some rats (the second cohort described in neural recording procedures), an additional camera was mounted in the back of the pellet receptacle to allow a close-up view of pellet delivery and retrieval. For rats that did not have this additional camera, pellet retrieval was visible from the side-view camera. The following behavioral events were rated only once at the beginning of the movement sequence using custom software (DataRat; The Aldridge Lab): orienting, head movement toward the lever CS during the CS period; lever sniff, tip of the nose coming in contact (or near contact, $<2 \mathrm{~mm}$ ) with the lever; lever press, deflection of the lever with front forepaws; lever bite, nibbling at the lever or grasping the lever between the teeth; nose in, the moment when the rat's nose entered the food receptacle either as part of a goal-tracking response or retrieval of the pellet (specific movements directed toward the food cup, such as biting or licking the food cup, were rarely observed and not counted separately); nose out, the moment when the rat removed its nose from the magazine and moved away from the magazine; and pellet-in-mouth, the moment when the rat retrieved the pellet with its mouth.

In addition to rating individual behaviors, we used video analysis to measure bouts of interaction with the lever or food cup. For each trial, the start of lever interaction was marked with a "lever start" label placed one frame (1/30 s) before the first instance of contact with the lever regardless of the type of contact (i.e., sniff, press, or bite). "Lever end" marked the moment when the rat moved away from the lever either with its front forepaws or entire body. If the rat did not leave the lever until the end of the trial, the lever end label was placed at the moment when the lever began to retract. We also marked the beginning of magazine interaction with a "magazine start" label. Because a bout of interaction with the magazine did not include several different types of behavior, magazine start corresponded to the first rating of "nose in" per trial. Magazine end marked the moment when the rat stopped goal tracking and moved away 
from the magazine. If rats kept their noses in the magazine throughout the trial and the pellet delivery phase, the moment of lever retraction was labeled as "magazine end." If a trial included multiple separate bouts of interaction with the lever or food cup, only one of each type was counted.

\section{Quantification of PCA behavior}

The tendency to display a ST or GT CR was determined by calculating the probability of contacting the lever or food cup in each trial. The probability difference score was defined as the number of trials with lever contact or food cup entry, divided by the total number of trials. Instances of lever sniff were considered contact with the lever even if they did not result in lever deflection. Probability difference was determined for each session, calculated as the probability of lever contact minus the probability of magazine contact. The resulting scores ranged from -1 to +1 , with -1 indicating a strong bias toward goal tracking, +1 indicating a strong bias toward sign tracking, and 0 indicating that responses were evenly distributed between the lever and magazine. For each rat, probability difference scores were averaged across the PCA sessions in which neural recordings were made. Rats with average scores of +0.5 or above were classified as STs, rats with average scores of -0.5 or below were classified as GTs, and rats with scores between +0.5 and -0.5 were classified as intermediates (INs).

\section{Electrodes and neural recording procedures}

Two varieties of custom-made electrode microdrives were used. The first had 16 Teflon-coated tungsten wires arranged in two individually moveable bundles of eight wires (four $25 \mu \mathrm{m}$ and four $50 \mu \mathrm{m}$; California Fine Wire Company) and signals were obtained with a wireless head stage and recording system (Triangle Biosystems). The second type of electrode had $3210 \mu \mathrm{m}$ nickel chromate wires (California Fine Wire Company) twisted into eight "tetrodes" of four wires each arranged in two individually movable bundles of four tetrodes each. The 32-channel electrodes were connected to an OmniPlex D neural data acquisition system (Plexon). Both types of microdrives had one channel designated as a reference wire. All electrodes were initially implanted $1 \mathrm{~mm}$ above the VP. On the day of the first recording session, bundles were lowered $1 \mathrm{~mm}$ to the top of the VP and, on subsequent recording days, bundles were lowered an additional $80-160 \mu \mathrm{m}$ to record from different cells each day.

One cohort of rats (2 STs, 5 GTs, 8 INs, and 2 unpaired) had 16channel electrodes implanted before PCA training. After 1 week of recovery, rats underwent $5 \mathrm{~d}$ of PCA training in which wireless head stages were attached to allow habituation, but neural activity was not recorded. Neural recordings started on PCA day 6 and continued for an additional 5-10 PCA sessions. At the beginning of recording sessions, rats had their cages placed near the chamber and then waited in their cages with the wireless head stage attached for 10-15 min while neural signals were examined for detected cells. Rats were then placed in the chamber and the session started after a $1 \mathrm{~min}$ habituation period. A second cohort of rats (4 STs, 3 GTs, 1 IN, 2 unpaired) had 5 d of PCA training before implantation of 32-channel electrodes. After recovering from surgery rats were retrained in the PCA task for $2 \mathrm{~d}$ and allowed to habituate to the attachment of the recording wire. Neural recordings began on PCA day 8 and continued for an additional 5-12 PCA sessions. Rats in the second cohort, but not the first cohort, had white noise on during PCA sessions. At the beginning of recording sessions, rats in the second cohort were tethered to the wire and waited $10-15 \mathrm{~min}$ in the chamber while neural signals were examined. The start of the session was signaled by the house light and white noise. These two cohorts did not differ in terms of behavior or neural responses during the PCA task, so their data were combined.

\section{Neural data analysis}

Neural activity was assessed in the periods $10 \mathrm{~s}$ before lever extension to $5 \mathrm{~s}$ after pellet delivery. The intertrial interval apart from the $10 \mathrm{~s}$ period before lever extension was not examined. Using Offline Sorter (Plexon), units were separated from each other and from background noise using spike waveform features and principal components analysis. Unit firing patterns were analyzed with Neuroexplorer (Nex Technologies) and custom software (Epochbuilder; The Aldridge Lab). Interspike intervals and autocorrelations were examined to insure the unitary nature of each discriminated unit. Cross-correlations were performed between all cells recorded in a session. If a single neuron was recorded on more than one channel, only the channel with the clearest waveform shape and best isolation from noise and from other units was used in the final analysis.

Responses to task events. For neural analysis, cue onset was defined as the moment that lever extension started. Cue offset was the moment that lever retraction began, which occurred simultaneously with the pellet feeder clicking sound. In the unpaired group, the click and US were separate from lever retraction because lever insertion and retraction was noncontingent with reward delivery. A pre-CS baseline period was defined as a period extending $10 \mathrm{~s}$ before lever extension onset. The cue period, during which sign-tracking or goal-tracking typically (but not always) occurred, was defined as the period from $1 \mathrm{~s}$ after cue onset until cue offset. Any trials in which the rat failed to perform any $\mathrm{CR}$ at all during the $8 \mathrm{~s}$ CS period were discarded (in $92.4 \%$ of sessions, the number of trials excluded was $\leq 2$ ).

Changes in neural activity relative to the pre-CS baseline were computed for four epochs: (1) CS onset, which is the epoch beginning with lever extension ( $0-0.4 \mathrm{~s}) ;(2)$ interaction interval, consisting of the last $7 \mathrm{~s}$ of the $8 \mathrm{~s}$ period when the lever was extended and rats engaged in ST and GT behavior (1-8 s); (3) CS offset/pellet delivery, which begins at the moment of lever retraction and has simultaneous activation of the food pellet feeder $(8-8.4 \mathrm{~s})$; (4) US, which is the interval when the reward pellet reached the food cup (8.6-9.6 s). For unpaired rats, the baseline period for pellet trials was the $5 \mathrm{~s}$ period immediately preceding pellet delivery. This baseline was used in unpaired rats for epochs 3 (pellet feeder click) and epoch 4 (US). To determine whether a cell was responsive to task events, we compared the firing rate (spikes/s) in each of the 4 epochs to the corresponding baseline with four Mann-Whitney $U$ tests (Bonferroni-corrected $\alpha=0.05 / 4$ ). Cells were considered responsive if they showed a significant increase or decrease in firing during that epoch.

Responses to behavioral action events. Neural activity was also examined in response to behavioral action events identified through video analysis. Neural correlates of lever start and magazine start actions (described in video analysis procedures) were assessed in $500 \mathrm{~ms}$ epochs -100 to 400 ms relative to the action onset. Responsiveness was assessed with a Mann-Whitney $U$ test comparison of a $1 \mathrm{~s}$ pre-event baseline $(-1.5$ to $-0.5 \mathrm{~s}$ ) to each epoch (Bonferroni-corrected $\alpha=0.05 / 2$ ).

Response coding. Responses of every unit were confirmed by visual inspection of perievent rasters and histograms to insure that spurious contributions in only a few trials were not the sole determinant of the response. Based on the statistical assessment of neural activation, responsive cells were counted and the proportions of responsive neurons were calculated. To determine the magnitude of firing, we calculated the normalized firing rate for each cell (following the same methods as Root et al., 2013). The firing rate (spikes/s) of each cell was normalized to baseline using the formula $\mathrm{B} /(\mathrm{A}+\mathrm{B})$, where $\mathrm{B}$ is each bin used in the analysis and $\mathrm{A}$ is the unit's baseline. This formula produces values ranging from 0 to 1 , with a value of 0.5 representing no change from baseline. This formula also preserves the directionality of a unit's response, with values closer to 1 reflecting excitation and values closer to 0 reflecting inhibition. In figures that display firing rate change, deviations from baseline were transformed by taking the absolute value of the deviation from 0.5 (i.e., |normalized firing rate $-0.5 \mid$ ). This formula shows the magnitude of change without directionality, with both excitation and inhibition shown as a positive change from baseline. In some analyses, neural firing is displayed as a heat map to depict overall population changes. In heat maps, each row represents the normalized firing rate of a single unit across the epoch described in the analysis in either 100 or $200 \mathrm{~ms}$ bins. Heat maps include all units recorded from each group whether they are classified as responsive to a particular event or not. Colors represent degree of deviation from 0.5 , with blue indicating inhibition of firing and red/yellow indicating excitation.

\section{Histology}

On the last day of recording, a current was passed through one wire on each bundle, creating an electrolytic lesion that marked the terminal position of the electrode tip. After 3-7 d, rats were killed by $\mathrm{CO}_{2}$ inhalation and brains were removed and rapidly frozen in isopentane. Brains were sectioned on a cryostat in 40 or $50 \mu \mathrm{m}$ coronal sections and stained 
with cresyl violet. Electrode placement was verified with light microscopy and drawn onto figures from a rat brain atlas (Paxinos and Watson, 2007). For six rats, one of the two electrode bundles fell outside the range of the VP and data obtained from these bundles were excluded. The placement of the remaining electrode bundles is shown in Figure 1, with the range of recording sites representing the dorsal-ventral depth at which usable cells were detected.

\section{Statistics}

Behavioral results were analyzed with a linear mixed model that included group as a fixed factor and time (days 6 or 8-14) as a covariate. Significant main effects of group were further analyzed with independent $t$ tests (Bonferroni corrected) comparing values averaged across all recording days for each animal. To examine the proportion of neurons responsive to a particular event, we conducted multiple pairwise $\chi^{2}$ tests comparing ST, IN, GT, and unpaired groups (Bonferroni corrected). To examine differences in the magnitude of firing among responsive neurons, we compared these four groups with one-way ANOVAs followed by Bonferroni-corrected post hoc tests. Outliers were identified and removed from analyses of magnitude using the ROUT method. In the above analyses, group n's represented the number of cells per group. Because there was considerable variation in the number of cells recorded from individual rats (ranging from 5 to 37 ), it is conceivable that the above results could have been heavily influenced by the rats with the most recorded cells. To address this possibility, we also examined neural activity in each rat separately and performed correlational analyses (Pearson's r), with $n$ 's representing a single data point per rat. Statistical procedures were performed with R (version 3.0.2), GraphPad Prism (version 6), and SPSS (version 22).

\section{Results}

Behavioral results: individual differences in PCA behavior During training, some rats acquired primarily ST CRs, others GT CRs, and others vacillated between the two, as we have described previously (Flagel et al., 2007; Meyer et al., 2012). The probability of contacting the lever was significantly higher in STs and INs than in GTs and the unpaired group (group: $F_{(3,176.9)}=17.1, p<$ 0.001 ; Fig. $2 A$ ), whereas the probability of contacting the food cup was greater in GTs and INs than STs and the unpaired group (group: $F_{(3,157.02)}=7.24, p<0.001$; Fig. $2 B$ ). These data were used to classify rats as STs, GTs, or INs, as described in the Materials and Methods, and the resulting groups (6 STs, 9 INs, 8 GTs, 4 unpaired) maintained their different patterns of conditioned responding during the sessions when electrophysiological recordings were obtained (Fig. $2 C$ ). Although CRs differed between groups, the median latency to orient to the CS did not differ among STs, INs, and GTs (Fig. 2D). The mean latency to make the initial contact with the target of conditioned approach differed significantly between groups $\left(F_{(2,94.24)}=4.87, p<0.01\right)$, with STs showing faster contact than INs and GTs (Fig. 2E).

Despite differences in approach latency, once rats contacted their respective targets the vigor with which they interacted with the target was similar for STs, INs, and GTs, as illustrated in
Sign Trackers
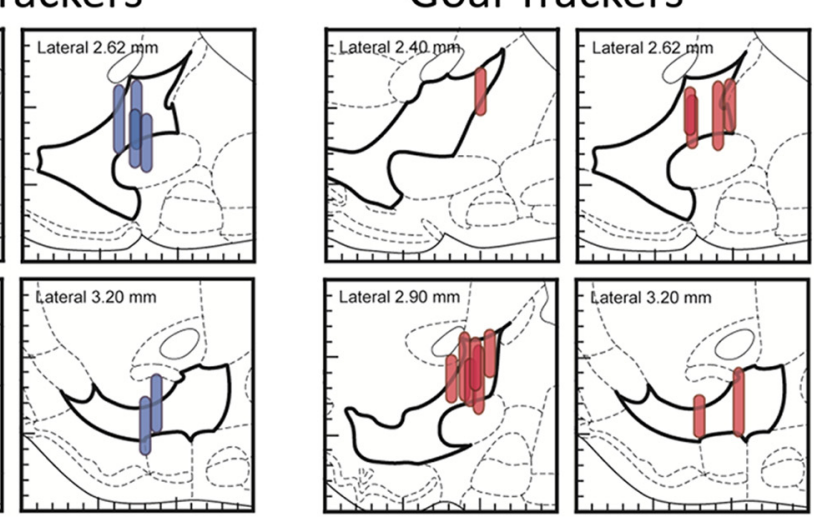

Goal Trackers

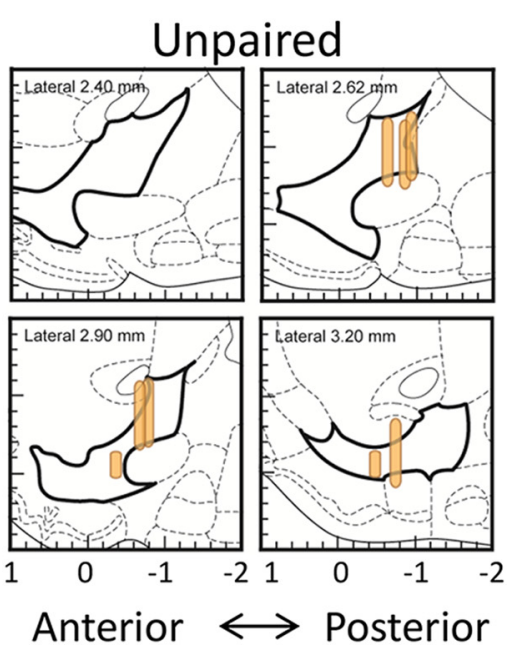

Figure 1. Recording sites in the VP are shown for each group. Vertical lines mark the dorsal-ventral range of recording sites from each wire bundle because wires were initially implanted above the VP and lowered 80-160 $\mu \mathrm{m}$ on each day of recording. Electrode placement is shown in the sagittal plane, with left and right hemispheres combined and bregma $=0$.

Movie 1. Contacts are not shown for every session because sniffing the lever was considered part of a lever-directed CR, but was not captured by counts of lever deflections. To further quantify the vigor with which rats engaged their respective targets, we counted the number of lever deflections and magazine entries (per second of interaction) in 10 randomly chosen trials from each group. All groups had similar numbers of contacts (STs lever contacts, $0.67 \pm$ 0.08; INs lever contacts, $0.68 \pm 0.11$; INs magazine entries, $1.07 \pm$ 0.18 ; GTs magazine entries, $0.77 \pm 0.17)$ that did not differ significantly between groups $\left(F_{(3,36)}=1.77\right.$, n.s. $)$. This further establishes that, although the behavior of STs and GTs is directed toward different targets, the topography of the behavior and vigor of interaction with those targets was very similar (also see the online video posted by Mahler and Berridge, 2009).

\section{VP neural activity was greater in STs during the period of cue exposure}

Of 339 cells recorded from STs, GTs, and INs, the majority $(85.7 \%)$ of VP neurons were responsive during the PCA task, but patterns of activity varied as a function of group (e.g., STs vs GTs). Overall group differences in firing rate changes across the duration of a trial are illustrated in Figure 3 and Figure 4 shows the percentage of neurons responsive to specific task events in GTs and STs. The most prominent feature was that, in STs, VP neurons showed much more sustained firing changes during the period when the lever CS was present than 

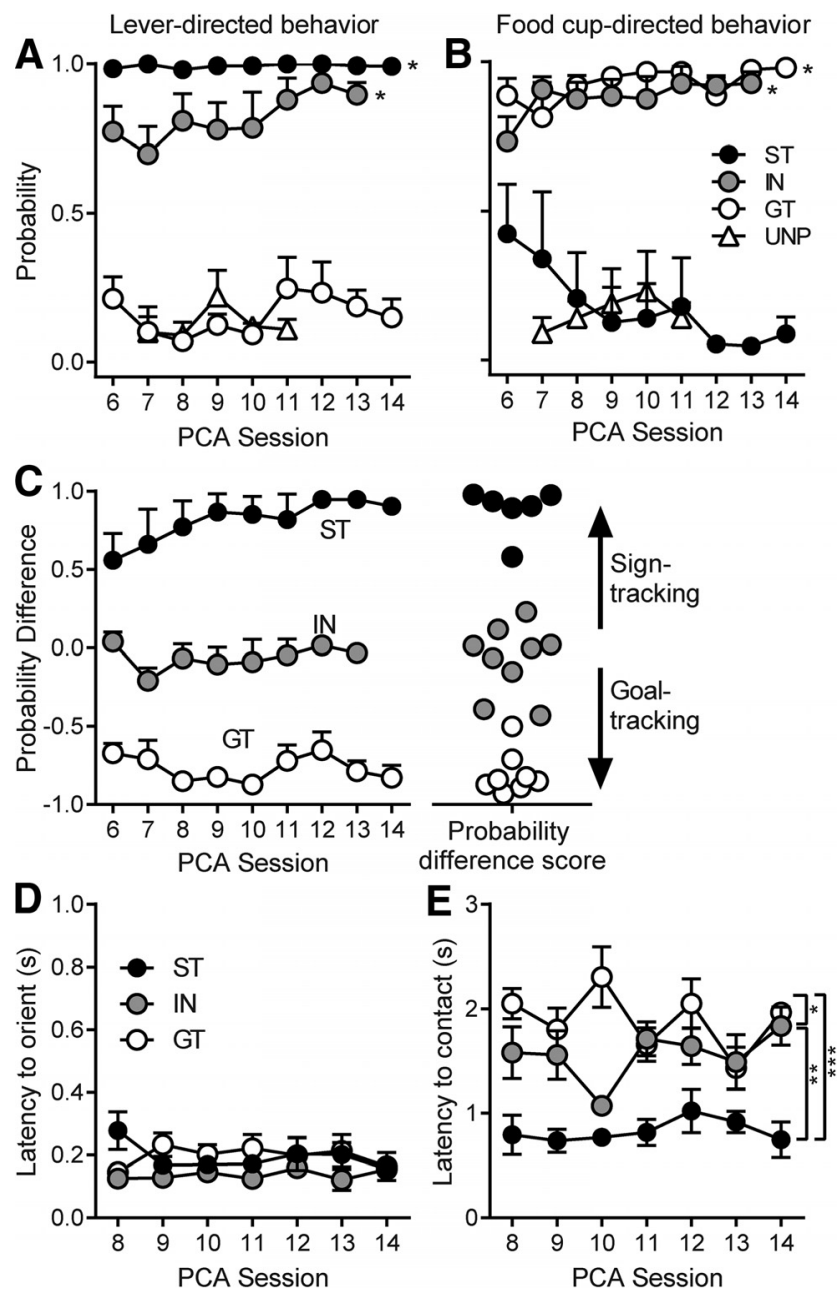

Figure 2. The probability of contacting the lever or food cup (mean \pm SEM) during the PCA sessions in which neural activity was recorded. Rats showed stable individual differences in the tendency to display ST or GT CRs during exposure to the lever CS. In the unpaired group (UNP), the lever was not paired with food reward. $A, B$, ST s and IN s had a higher probability of contacting the lever than GTs and the unpaired group $\left({ }^{*} p<0.001 ; \boldsymbol{A}\right)$, whereas GTs and INs had a greater probability of contacting the food cup than STs and the unpaired group $\left({ }^{*} p<0.001 ; \boldsymbol{B}\right)$. C, Probability difference (the probability of lever contact minus the probability of food-cup contact) was used to classify rats as STs, INs, or GTs. Probability difference scores were calculated for each rat by averaging across all days of recording and rats were classified as STs if their scores fell $>0.5$, GTs if their scores fell below -0.5 , and INs iftheir scores were between 0.5 and -0.5 . D, Therewere no differences between STs, INs, and GTs in the latency to display an orienting response to the lever CS. $\boldsymbol{E}$, Latency to make initial contact with the target of conditioned approach was shortest in STs, though INs were faster than GTs; ${ }^{*} p<$ $0.05,{ }^{* *} p<0.01,{ }^{* * *} p<0.001$.

in GTs, INs, or the unpaired control group. All groups showed phasic peaks at the onsets and offsets of the cue and at reward delivery. Although there were also group differences in response to these events (see detailed analysis below), they were less pronounced than the sustained responses seen during the CS period (Fig. 3).

Most VP neurons responded to multiple events (Fig. 4A). Compared to ST cells, GT cells showed more phasic responses to CS onset, CS offset, and/or consumption of the US, but were less responsive during the CS period. ST responses had more sustained contours of activation, whereas GTs had more punctate profiles. These properties were apparent on individual neurons in STs (each row in the heat map of Fig. $4 B$ ) as more intense and sustained increases and decreases. In contrast, neural activity in GTs stood out at cue onset, offset, and reward events on individual neurons (Fig. 4B). INs had an intermediate profile.
Proportion and magnitudes of neural responses to individual task events

For each of the task events, we examined neural representation by assessing the proportion of cells showing excitatory and inhibitory responses (a population code), as well as the magnitude of the change in neural firing (rate coding).

\section{CS onset}

CS onset was defined as the moment when the illuminated lever CS extended into the cage. This cue elicited a conspicuous, phasic increase in neural firing in all groups (STs, INs, and GTs), except the unpaired group, in which presentations of the lever/ light cue and rewards were explicitly unpaired (Fig. 3). Responses to CS onset may have been influenced by orientation to the cue because this movement typically occurred within the CS onset epoch. The ST, IN, and GT groups all showed a similar orienting response to the CS before approaching either the lever CS or the food cup (Fig. 2D; see also Yager and Robinson, 2013), which may have contributed to similarities in the neural responses to CS onset.

STs and INs both had a significantly larger proportion of cells responsive to CS onset than GTs $\left(\chi^{2}=9.28-14.04, p<0.05\right.$ $0.01)$ and all paired groups (STs, GTs, and INs) had more responsive cells than the unpaired group $\left(\chi^{2}=8.97-32.52, p<0.05-\right.$ 0.001 ; Fig. $5 A$ ). When the proportions of excitatory responses in STs, INs, and GTs were compared with each other, only INs and GTs differed significantly $\left(\chi^{2}=17.39, p<0.001\right.$; Fig. $\left.5 A\right)$. There were no group differences in the proportion of inhibitory responses, including when STs, INs, and GTs were compared with the unpaired group $\left(\chi^{2}=0.17-2.99\right.$, n.s.; Fig. 5A). Among cells that showed excitatory responses to CS onset, the magnitude of excitation was highest in STs. There was a significant group effect $\left(F_{(2,101)}=4.97, p<0.01\right)$ and post hoc tests showed that magnitudes were significantly higher in STs than INs $(p<0.01)$, but did not differ between STs and GTs (Fig. $5 B$ ). The magnitude of inhibitory responses did not differ between groups $\left(F_{(2,27)}=\right.$ 1.52 , n.s.). We also assessed correlations between the propensity to sign-track or goal-track (i.e., probability difference scores) and neural responses to CS onset. The relationship between probability difference scores and proportion of responsive cells approached significance ( $r=0.41, p=0.055, n=23$; Fig. $5 C$ ); however, there was a significant correlation between probability difference scores and the overall change in firing rate (median absolute rate change) $(r=0.42, p<0.05, n=23$; Fig. $5 D)$. Responses to CS onset are shown in heat maps in Figure $5 E$.

\section{Cue period}

The most robust group differences in neural activity occurred during the period when the lever CS was present, which we defined here as the last $7 \mathrm{~s}$ of the $8 \mathrm{~s}$ interval after the lever CS was extended into the cage. This is the period in which rats were engaged in ST or GT behavior and, during this period, STs showed substantially greater neural activity than both the INs and GTs (Fig. 3).

STs had approximately twice the number of responsive cells as INs or GTs during the cue period $\left(\chi^{2}=24.88-28.44\right.$, $p<0.001$; Fig. 6A). Furthermore, STs had more excitatory responses than INs $\left(\chi^{2}=9.19, p<0.01\right)$ and GTs $\left(\chi^{2}=11.25\right.$, $p<0.01)$ and more inhibitory responses than INs $\left(\chi^{2}=8.68\right.$, $p<0.05)$ and GTs $\left(\chi^{2}=9.31, p<0.05\right)$ (Fig. 6A). GTs and INs did not differ from each other on any of these measures. Notably, there were no responsive neurons in the unpaired group during the cue interaction period. The magnitude of 
excitation was also greater in STs compared with the other groups. There was a significant group effect in the normalized firing rate of excitatory cells $\left(F_{(2,99)}=11.79, p<0.001\right)$ and post hoc tests showed that magnitudes were significantly higher in STs compared with both INs $(p<0.01)$ and GTs $(p<0.001)$ (Fig. 6B). However, there were no significant group differences in the magnitude of inhibitory responses $\left(F_{(2,37)}=0.60\right.$, n.s. $)$. When we examined neural firing with respect to behavior of individual rats, we found that heightened responsivity during the cue period was positively correlated with the tendency to sign-track. Probability difference scores were significantly correlated with the proportion of cells responsive during the cue period $(r=0.61, p<0.01, n=$ 23; Fig. $6 C$ ), as well as the median firing rate change of cells recorded from individual rats $(r=0.71, p<0.001, n=23$; Fig. $6 D)$. The intensity of firing is also shown in heat maps, which were sorted by highest to lowest normalized firing rate during the $7 \mathrm{~s}$ cue period (Fig. $6 E$ ). Together, these results suggest that there is a strong relationship between the propensity to attribute incentive salience to a food cue and VP activity during exposure to that cue.

\section{CS offset/pellet delivery click}

A clear and distinct neural response in the VP was evoked in association with the pellet dispenser clicking sound, which occurred simultaneously with the moment that the lever CS began its retraction into the cage wall, which took $330 \mathrm{~ms}$ to withdraw fully. This third epoch was defined as the $400 \mathrm{~ms}$ period immediately after activation of the pellet dispenser. This event was separate from (and preceded) the arrival of the pellet into the food cup, which took, on average, $600 \mathrm{~ms}$ to reach the food cup after the dispenser click. During this CS offset/pellet delivery click epoch, the unpaired group showed a vigorous increase in firing, in contrast to the CS onset cues and the cue interaction periods, during which unpaired animals exhibited little or no neural activation. The audible click of the pellet dispenser likely functioned as a predictive Pavlovian CS in the unpaired group.

There were no group differences in the proportion of cells responsive to the CS offset/pellet delivery cue $\left(\chi^{2}=0.13-3.33\right.$, n.s.) regardless of whether those responses were excitatory $\left(\chi^{2}=0.07-3.06\right.$, n.s. $)$ or inhibitory $\left(\chi^{2}=0.01-5.06\right.$, n.s.; Fig. 7A). There was a significant group effect for the magnitude of excitatory responses to the CS offset/pellet delivery cue $\left(F_{(3,98)}=4.53, p<0.01\right)$; however, post hoc tests showed that STs had a significantly higher magnitude only when compared with INs $(p<0.01)$ and not when compared with GTs or the unpaired group (Fig. 7B). Again, no differences were found in the magnitude of inhibitory responses $\left(F_{(3,60)}=1.72\right.$, n.s. $)$. Neither sign-tracking nor goal-tracking behavior seemed to be an important differentiator of neural activation patterns at this time because there was no significant correlation between probability difference scores and the proportion of responsive cells ( $r=0.25$, n.s., $n=23$ ) or the median firing rate change for individual rats $(r=0.04$, n.s., $n=23)$.

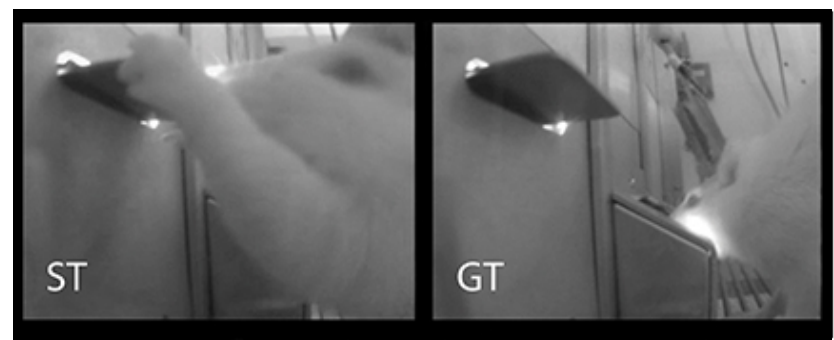

Movie 1. Examples of the CRs of GTs, STs, and INs.

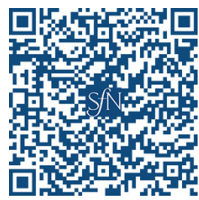

\section{US consumption}

The fourth peak in neural activity occurred during the moment when rats retrieved the pellet from the food magazine. This US epoch was defined as the interval from 0.6 to $1.6 \mathrm{~s}$ after the pellet delivery click. Although close in time to CS offset/pellet delivery period, the activation associated with US was characterized by a separate and distinct peak of activity that overlapped with pellet retrieval (in $>90 \%$ of trials, the pellet was retrieved within this $1 \mathrm{~s}$ interval).

In all groups (STs, GTs, INs, and unpaired group), VP neurons were strongly activated by reward receipt. There were no group differences in the total proportion of responsive cells $\left(\chi^{2}=0.05-1.55\right)$; however, when responses were broken down into excitations and inhibitions, we found that STs and INs had more excitations and fewer inhibitions than the unpaired group. For excitations, STs and INs both had significantly more responses than the unpaired group $\left(\chi^{2}=10.94-\right.$ 11.02, $p<0.01$ ), but there were no significant differences between STs, INs, and GTs. For inhibitions, STs and INs had significantly fewer responses than the unpaired group $\left(\chi^{2}=\right.$ $7.76-9.62, p<0.05)$, but, again, there were no significant differences among STs, INs, and GTs (Fig. 7C). The magnitude of excitatory responses was significantly higher in STs than in

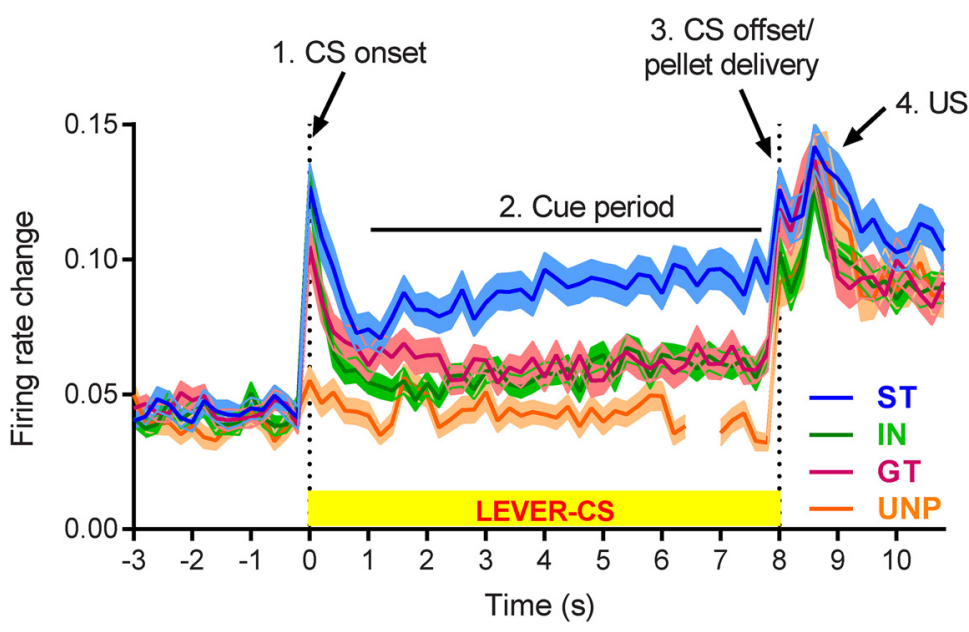

Figure 3. Average VP firing rate changes across a trial. VP cells were very responsive to task events in STs, GTs, and INs (but not the unpaired control group; UNP). During the cue period, when the lever was available and rats performed ST or GT (Rs, the change in neural firing was greater in STs than in GTs or INs. All groups, including the unpaired group, showed enhanced neural activity in response to pellet delivery and receipt. The UNP line is shown with a gap between lever and pellet trials because the CS and US occurred in separate trials for the unpaired group. Data are shown as normalized firing rates (mean $\pm \mathrm{SEM}$ ), with all change from baseline shown as positive in direction. All cells from each group are included, whether they were considered responsive to task events or not (ST, $n=108$; IN, $n=112 ; \mathrm{GT}, n=116 ; \mathrm{UNP}, n=63$ ). 
A

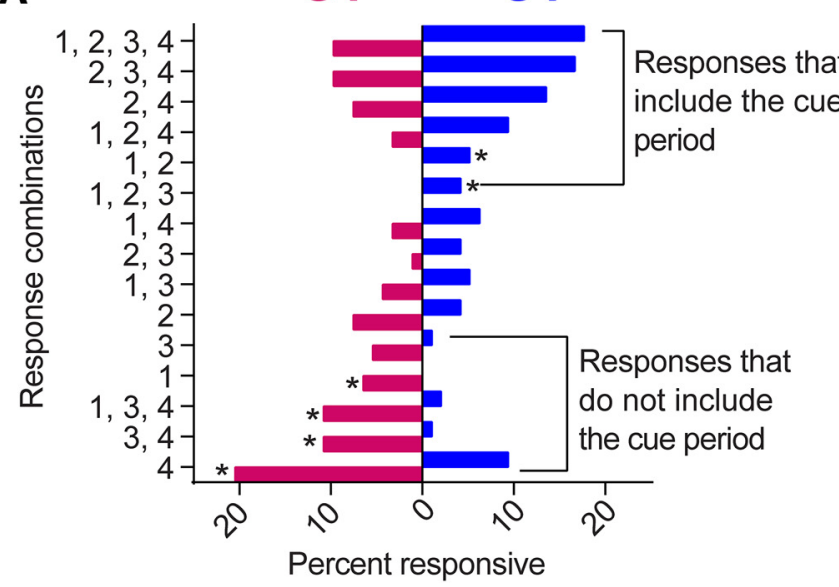

B
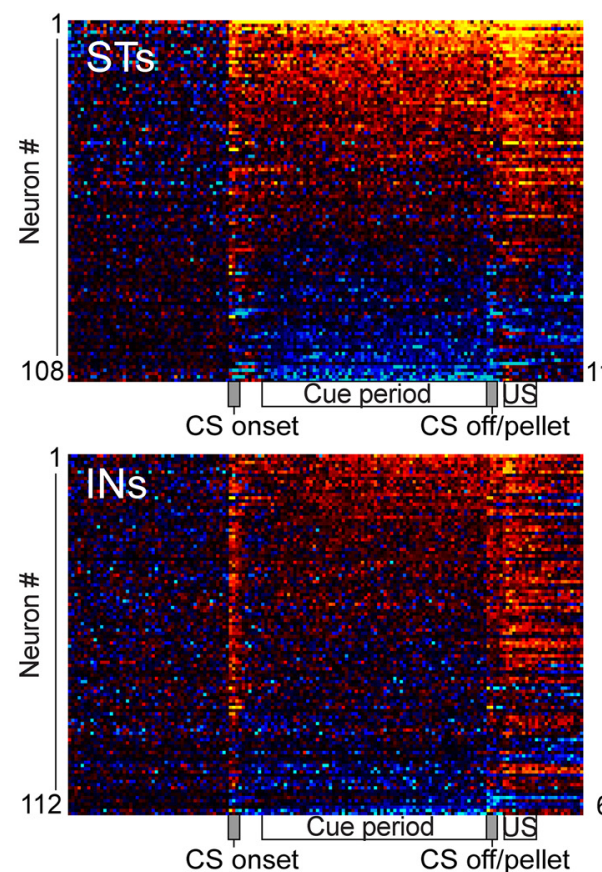

Normalized Firing Rate

$\begin{array}{llll}0.2 & 0.4 & 0.6 & 0.8\end{array}$ Inhibition $0.2-0.4+0.6+0.8$ Excitation
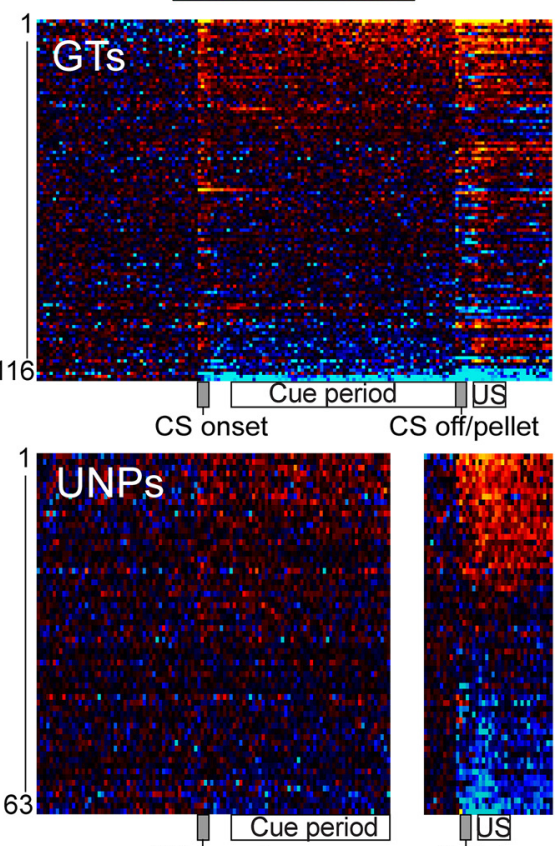

CS onset

Figure 4. Many VP cells were responsive to more than one of the four task events and several different response combinations were observed. $A$, Percentage of cells with each combination of responses. The most frequent firing patterns for STs included the cue period in combination with other CS and/or US events, whereas GTs frequently responded to combination of CS onset, CS offset, and/or US retrieval, but not the cue period $\left({ }^{*} p<0.05\right.$, significant differences between STs and GTs, $\chi^{2}$ with uncorrected $p$-values). $B$, STs have more sustained changes in activity compared with the punctate pattern in GTs. Overall VP activity is depicted for each group in heat maps, in which each row represents the firing of an individual cell, with rows sorted by highest to lowest normalized firing rate during all four events combined.

INs and GTs. There was a significant group effect for magnitude $\left(F_{(3,189)}=7.11, p<0.001\right)$ and significant post hoc differences when STs were compared with GTs $(p<0.001)$ and INs $(p<0.01)$, but there was no significant difference when the STs were compared with the unpaired group (Fig. $7 B$ ). Again, there were no significant group differences in the magnitude of inhibitory responses $\left(F_{(3,52)}=2.18\right.$, n.s. $)$. Probability difference scores were not significantly correlated with the percentage of responsive units from each rat $(r=-0.04$, n.s., $n=23)$. There was also no significant correlation between probability difference scores and the median firing rate change of cells from each rat $(r=0.19$, n.s., $n=23)$, which is in

\section{CS onset \\ 2. Cue period \\ 3. CS offset/ pellet delivery 4. US}

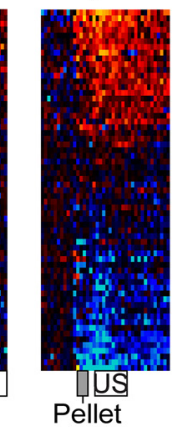

contrast to the group differences in magnitude described above. Because the US epoch occurred so close in time to the CS offset/pellet delivery epoch, both are shown in the same heat maps in Figure $7 D$.

\section{Neural responses associated with behaviorally defined events}

In addition to the analysis of experimenter-determined task events above, we also evaluated the neural correlates of ST and GT behavioral actions identified in a frame-by-frame video analysis during the cue interaction period. For this analysis, we used the lever start, lever end, magazine start, and magazine end actions, as defined in the Materials and Methods. We excluded trials with no CRs and sessions that had $<10$ actions to assess. For INs, sessions were required to have at least 10 lever events and 10 magazine events and, if both lever and magazine interaction occurred within a single trial, both events were counted. If a trial included multiple bouts of interaction, the first instance of each type was counted as long as the duration was at least $400 \mathrm{~ms}$. The number of cells assessed were as follows: STs-Lever, $n=101$; INs-Lever, $n=88$; INsMagazine, $n=88$; and GTs-Magazine, $n=114$.

\section{Lever and magazine interaction intervals}

During the CS period, rats were typically engaged in sign-tracking or goal-tracking behavior; however, there was always some variation in behavior from one trial to the next. For example, even among strong STs and GTs (meaning those with probability difference scores close to +1 or -1 , respectively), there were trials in which the start of the CR was delayed by a few seconds, there were occasions when STs went to the magazine or GTs went to the lever, and the latency to begin the CR differed between groups. Therefore, we isolated the neural activity that occurred specifically during the periods when STs and GTs were performing their respective dominant CRs and excluded the moments when they were engaged in any other type of behavior. Within each trial, we identified lever interaction intervals, defined as the time between lever start and lever end, and magazine interaction intervals, defined as the time between magazine start and magazine end (Fig. 8A). To determine responsiveness, the normalized firing rates within these intervals were compared with the pre-CS baseline firing from the same trial.

We found that STs had greatly enhanced neural activity while interacting with the lever CS compared with GTs interacting with the food magazine. STs also had enhanced activity compared with INs regardless of whether the INs were engaged with the lever or with the magazine. STs had a very high percentage of cells 
responsive during the interval of lever interaction (80\%). In contrast, INs and GTs had significantly fewer cells responsive during the performance of their respective CRs $\left(\chi^{2}=28.02-44.3, p<0.001\right)$ and there was no significant difference between IN-Lever and IN-Magazine responses $\left(\chi^{2}=2.14\right.$, n.s.; Fig. $\left.8 B\right)$. STs had significantly more excitatory responses than INs and GTs $\left(\chi^{2}=10.3-19.49, p<\right.$ 0.01-.001), as well as more inhibitory responses than IN-Lever $\left(\chi^{2}=7.03, p<\right.$ $0.05)$, IN-Magazine $\left(\chi^{2}=9.92, p<0.01\right)$, and GTs $\left(\chi^{2}=8.84, p<0.05\right)$. Within INs, there were no significant differences between IN-Lever and INMagazine responses among excitations $\left(\chi^{2}=1.56\right.$, n.s. $)$ or inhibitions $\left(\chi^{2}=\right.$ 0.31 , n.s.) (Fig. $8 B$ ). The magnitude of excitation was significantly greater in STs, with a significant group effect $\left(F_{(3,102)}=7.54, p<0.001\right)$ and a significant post hoc difference between STs and GTs $(p<0.001)$ and between STs and IN-Lever $(p<0.05)$, but not between STs and IN-Magazine, or between IN-Lever and IN-Magazine (Fig. 8C). Probability differences scores were significantly correlated with the proportion of responsive cells $(r=0.44, p<$ $0.05, n=28$ ) and the median firing rate change of cells from each rat $(r=0.64$, $p<0.001, n=28$; Fig. $8 D, E)$.

Initial contact with the lever or food cup There were phasic neural activations at the moment of initial contact with the lever or magazine (i.e., lever start or magazine start). These responses were typically seen in neurons that were also responsive to other task events; however, these contact activations appeared to be separate, with many cells showing distinct phasic peaks of activity when trials were aligned to lever or magazine start events (Fig. 9A). Responsiveness was determined by comparing spikes/s during the initial contact event (500 ms) with a baseline period that immediately preceded the event. We found that more cells were responsive to lever start in STs than were responsive to lever or magazine start in INs and GTs $\left(\chi^{2}=8.34-21.91, p<0.05-0.001\right)$ and that INs showed no significant difference between lever start and magazine start responses $\left(\chi^{2}=0.89\right.$, n.s. $)$. When responses were separated into excitations and inhibitions, STs had significantly more excitatory responses $\left(\chi^{2}=7.82-17.05, p<\right.$ $0.05-.001)$, but not more inhibitory responses $\left(\chi^{2}=0.28-2.75\right.$, n.s.) than INs and GTs. Again, INs showed no significant differences between lever and magazine start, either for excitations or
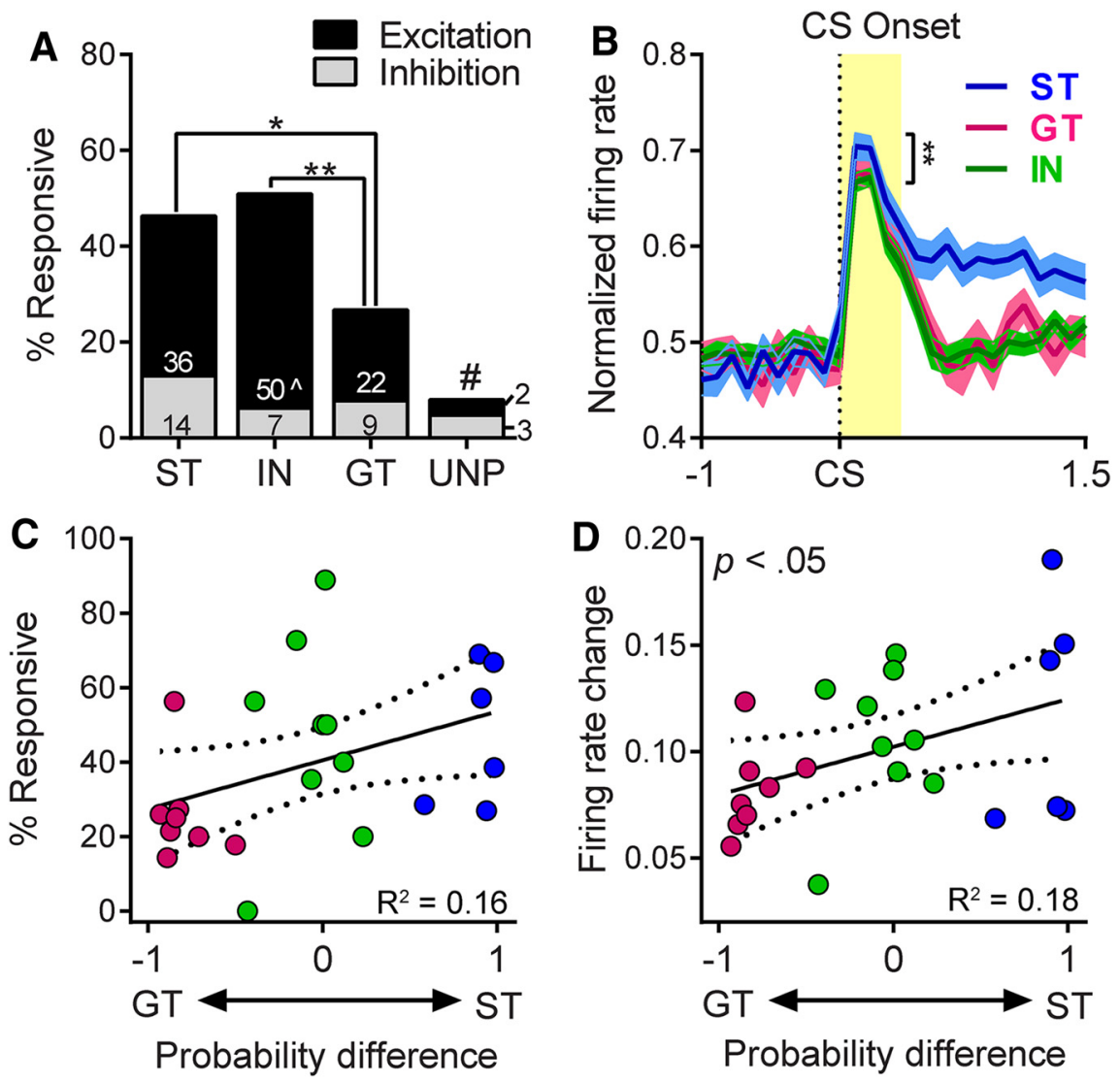

Probability difference

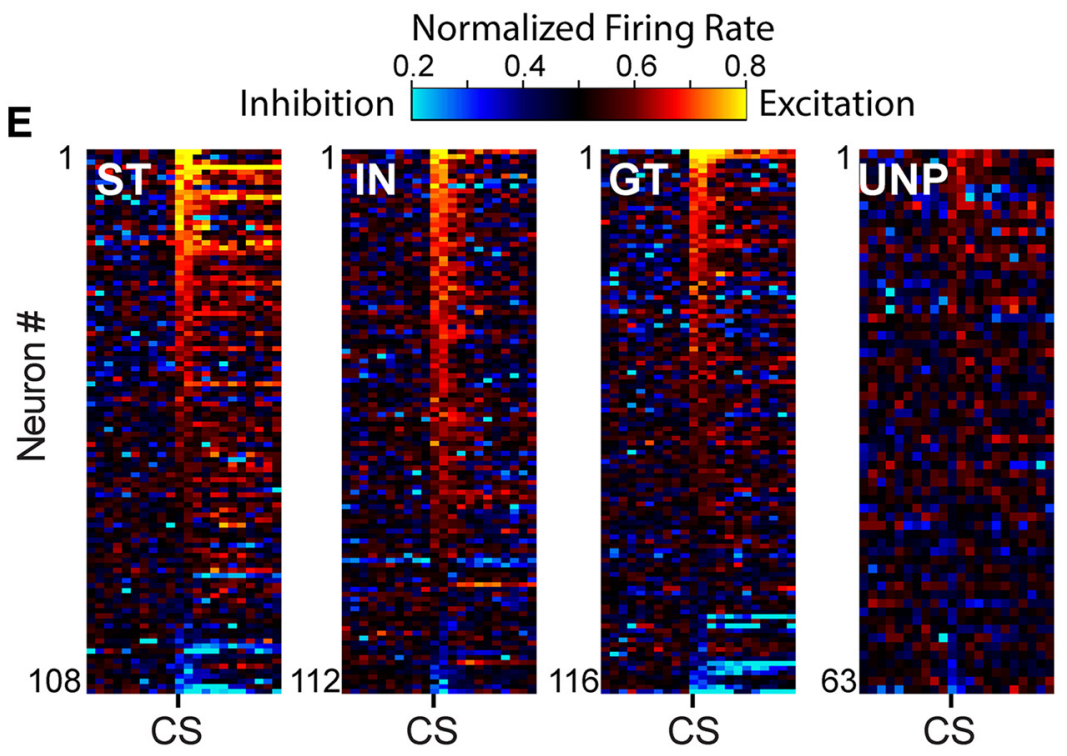

Figure 5. Neural activity during the cue onset period, defined as the first $400 \mathrm{~ms}$ after the lever $C S$ was extended into the cage. Presentation of the lever CS elicited an immediate (and largely excitatory) neural response in the VP.A,STs, GTs, and INs all had significantly more responsive cells than the unpaired (UNP) group, \#p <0.01-0.001; STs and INs both had significantly more responsive cells than GTs; ${ }^{*} p<0.05,{ }^{* *} p<0.01$. When excitatory responses were examined separately, the only significant difference was between INs and GTs, $\wedge p<0.001$. B , The magnitude of excitation did not differ between STs and GTs, although it was significantly greater in STs compared with $\mathrm{INs}^{* *} p<0.01$. C, D, When firing was examined in individual rats there was no correlation between probability difference scores and the percentage of responsive neurons (C); however, there was a significant correlation between probability difference scores and the overall magnitude offiring changes during $(S$ onset $(\boldsymbol{D})$. $\boldsymbol{E}$, Cue responses of individual cells are shown in heat maps ( $100 \mathrm{~ms}$ bins) sorted by highest to lowest firing rate during the first $200 \mathrm{~ms}$ after lever insertion cue onset. inhibitions $\left(\chi^{2}=0.04-2.10\right.$, n.s.; Fig. 9B). The majority of cells that were responsive to behavioral events were also responsive to task events, suggesting that most of these contact-responsive cells did not belong to a separate population that was distinct from 

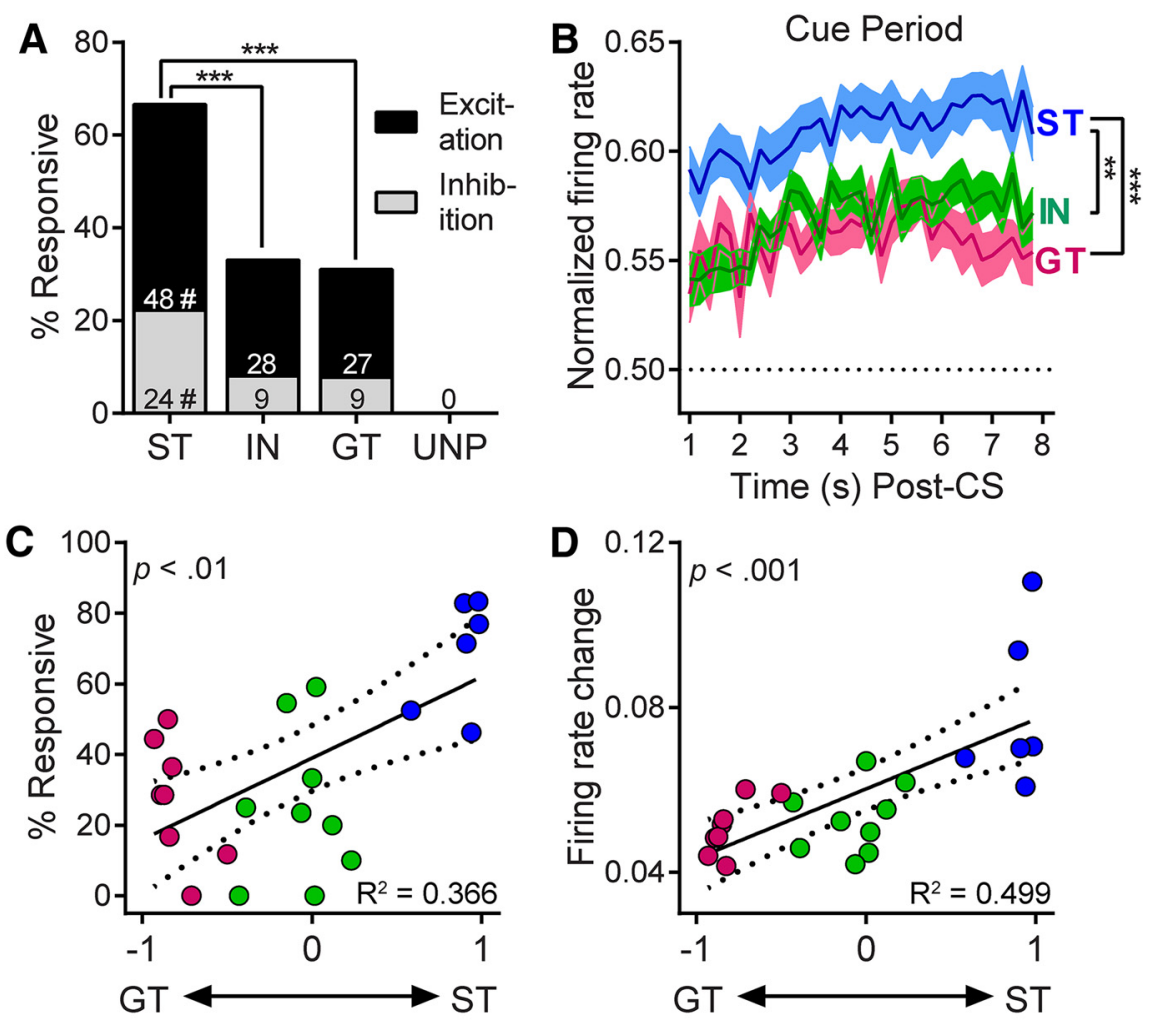

Probability difference

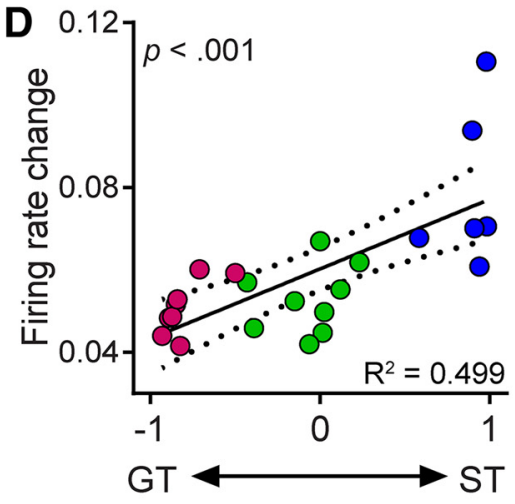

Probability difference

Normalized Firing Rate

$\begin{array}{llll}0.2 & 0.4 & 0.6 & 0.8\end{array}$
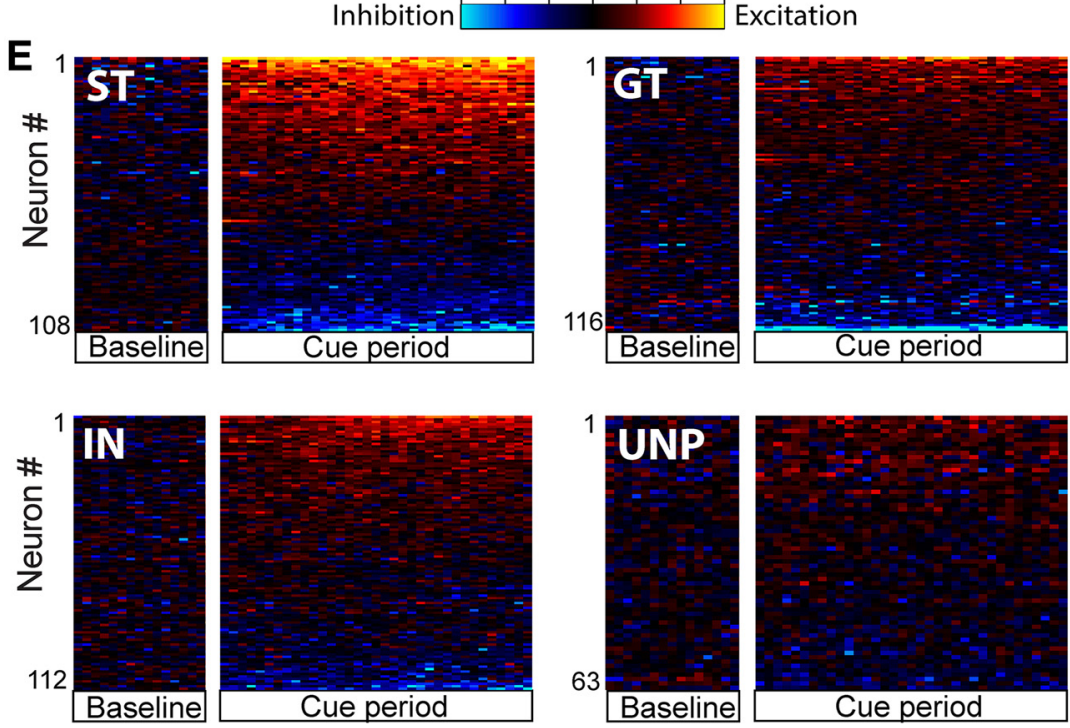

Figure 6. Neural activity during the cue interaction period while rats engaged in ST or GT behavior. $\boldsymbol{A}$, STs had significantly more responsive cells than GTs and INs; ${ }^{* * *} p<0.001$; and this was true for excitatory responses as well as inhibitory responses, \#p $<0.05-.01$. The unpaired group had zero cells that were responsive during the cue period. $\boldsymbol{B}$, Magnitude of excitation during the cue period was significantly greater in STs than in INs and GTs (the horizontal line represents baseline firing); ${ }^{* *} p<0.01,{ }^{* * *} p<0.001 . \boldsymbol{C}, \boldsymbol{D}$, When neural activity was examined for individual rats, probability difference scores were significantly correlated with the proportion of cells responsive during the cue period ( $\boldsymbol{C}$ and the magnitude of firing rate change during the cue period $(\boldsymbol{D})$. $\boldsymbol{E}$, Heat maps showing the firing patterns of individual neurons sorted by response magnitude during the cue period (shown in $200 \mathrm{~ms}$ bins). Firing is aligned to CS onset, but the CS onset interval is omitted. VP neural activity was visibly more intense for STs than it was for INs, GTs, or unpaired (UNP) rats.

trial-responsive cells (Fig. 9C). A small percentage of cells (5.9\% in STs and $1.8 \%$ in GTs) were primarily responsive to contact events and not to CS onset or the cue period; however, for the majority of contact-responsive cells there was substantial overlap with task events. In STs, for example, $74.4 \%$ of cells that responded to lever or magazine start continued to respond throughout the rest of the cue period. The intensity of VP neural responses to lever and magazine start is shown in heat maps in Figure $9 D$, with firing of individual cells aligned to the moment of the first lever or magazine contact and the average magnitude of excitatory responses shown above. There was a significant group effect for the magnitude of excitatory responses $\left(F_{(3,69)}=2.77, p<\right.$ 0.05 ), but there were no significant post hoc differences between groups after correction for multiple comparisons. There was no significant group effect for the magnitude of inhibitory responses $\left(F_{(3,17)}=2.1\right.$, n.s. $)$.

\section{Lever versus magazine responses}

in intermediates

One potential concern was that differences in neural activity between STs and GTs could be related to differences in motor activity associated with the two CRs. It should be noted, however, that the lever and magazine start events described above occurred immediately before contact with the respective targets and therefore could not have been influenced by any subsequent differences in motor activity. In addition, as mentioned above, INs showed no significant differences between lever and magazine interaction periods or between lever and magazine start events. We further examined the neural activity of INs and found that responses were similar whether rats contacted the lever or the magazine even when the two actions occurred within a single trial. Few cells responded to lever start or magazine start alone; instead, the majority of cells responded to a combination of both events and/or to trial events such as CS onset (Fig. 9E). Within the individual cells that showed excitatory responses to both lever start and magazine start $(n=9)$, there was no significant difference in the magnitude of excitation between the two events (paired $t$ test: $t_{(8)}=1.85$, n.s.; Fig. $9 F$ ).

Only responses to lever start and magazine start are shown, but similar results were found for lever and magazine interaction intervals. Of the 42 IN cells that were responsive during either lever or magazine interaction intervals, 13 (31\%) were responsive to both. Among the cells that showed excitatory responses to both $(n=12)$, there was no significant difference in the magnitude of excitation (paired $t$ test: $t_{(11)}=0.53$, n.s.). For INs, the baseline periods of lever and magazine start epochs could have potentially overlapped with lever or magazine interaction intervals that oc- 


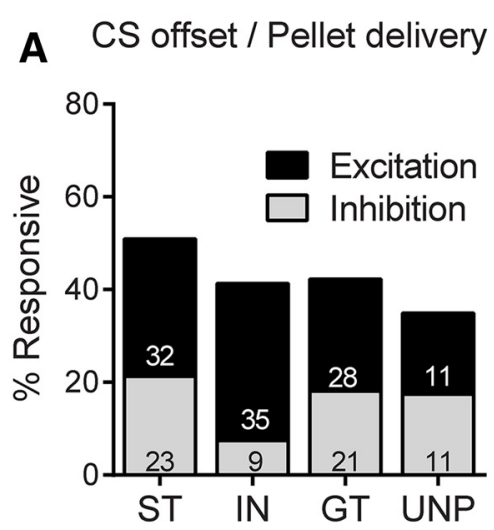

D

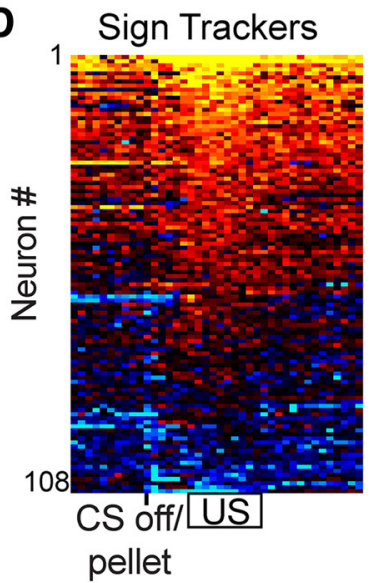

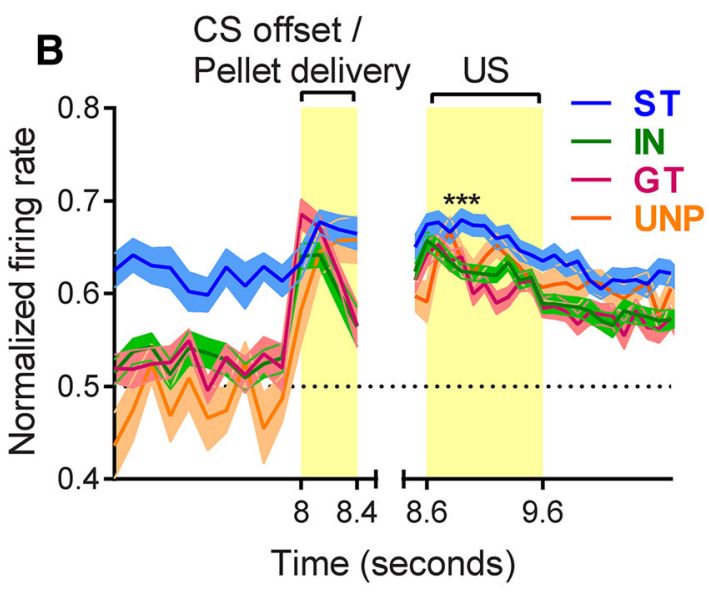

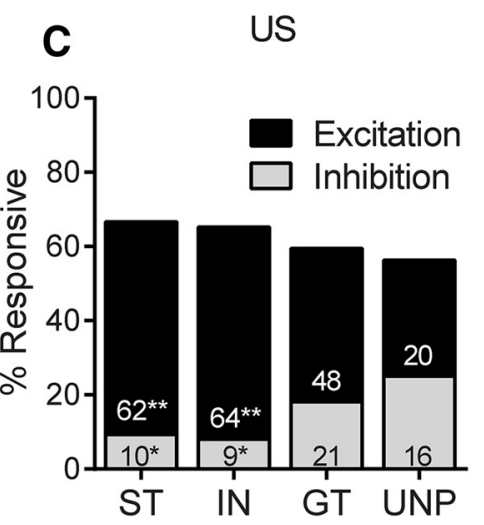

Figure 7. Neural activity during the moment when the pellet was delivered (CS offset/pellet delivery) as well as during the interval when the pellet was retrieved (US). A high percentage of VP neurons responded during this period, although the excitatory response to the US was greater in STs than in the other groups. $\boldsymbol{A}$, Percentage of neurons that responded to $C S$ offset/pellet delivery did not differ between groups. $\boldsymbol{B}$, Magnitude of excitatory responses to both the CS offset/pellet delivery epoch and the US epoch in 100 ms bins STs showed significantly higher magnitude of excitation during the US period compared with INs $(p<0.01)$ and GTs $(p<0.001)$. C, During the US epoch, STs and INs had more excitatory responses and fewer inhibitory responses than the unpaired (UNP) group; ${ }^{*} p<0.05,{ }^{* *} p<0.01$. D, Because the US epoch occurred so close in time to the CS offset/pellet delivery epoch, both are shown in the same heat maps, with rows sorted by the magnitude of firing during the US epoch.

curred earlier in the same trial. This potential overlap did not have a major effect on the results, however; because we found that 93.2\% of IN cells had the same response pattern whether they were compared with the pre-event baseline or a $10 \mathrm{~s}$ pre-CS baseline (excluding cells that had a sustained response during the $7 \mathrm{~s}$ cue period).

Finally, we examined whether cells were responsive to individual instances of lever bite and lever press events determined from video ratings (first instance of each per trial). We examined cells that had at least 10 instances of the event ( 99 from STs and 59 from INs) and found that only $2.5 \%$ ( 4 cells) could be considered responsive to either lever bite or lever press (with very small magnitude responses). The lack of a clear population response to these individual actions supports the idea that VP firing reflects motivational states rather than motor activity (see also Tindell et al., 2005).

\section{Discussion}

Individual variation in the form of the CR to a food cue (ST vs GT), which predicts the degree to which the CS is attributed with incentive salience, was reflected in neural activity in the VP. Specifically: (1) a large proportion of VP neurons were responsive to one or more task events $(85.7 \%)$; (2) more VP neurons were responsive to cue onset in STs than GTs (i.e., greater population code) and the magnitude of the change in firing rate to cue onset was positively correlated with the attribution of incentive salience to the lever CS (i.e., firing rate code); (3) during the cue period, there were approximately twice as many responsive neurons in STs than in GTs or INs, STs showed a greater sustained change in firing rate during this period, and both the number of responsive neurons and firing rate change was positively correlated with the degree to which rats were attracted to the lever CS; (4) group differences during the CS interaction period were especially pronounced when the data were analyzed in relation to behaviorally defined events, which takes into account trial-to-trial variation in the timing of behavior; and (5) finally, all groups showed a large response after CS offset (lever retraction and pellet delivery). Although the VP has been shown to be necessary for the acquisition of a ST, but not GT, CR (Chang et al., 2015), the current study establishes that neural activity in the VP also varies during the performance of ST and GT CRs.

Predictive value versus incentive value of the cue In this task, the lever is an equally effective CS in STs and GTs, evoking a CR in both. STs and GTs learn the CS-US relationship equally well, it is just the form of the CR that differs, reflecting the degree to which the lever CS is attributed with incentive salience 
A Sign Tracker

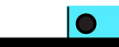
LEVER - CS

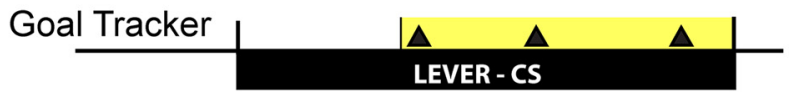

Intermediate

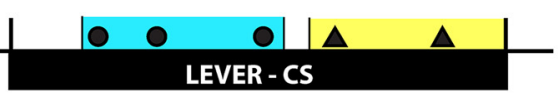

B

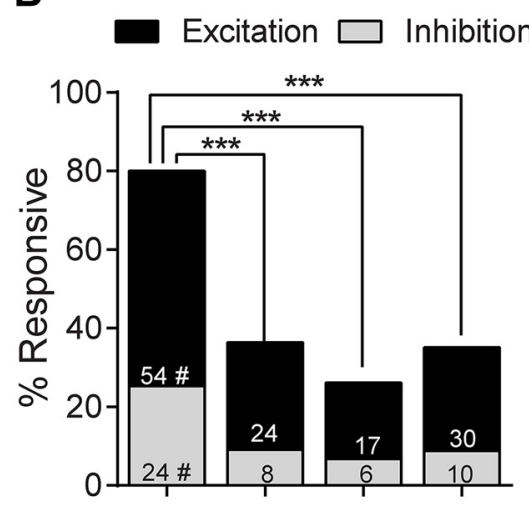

C

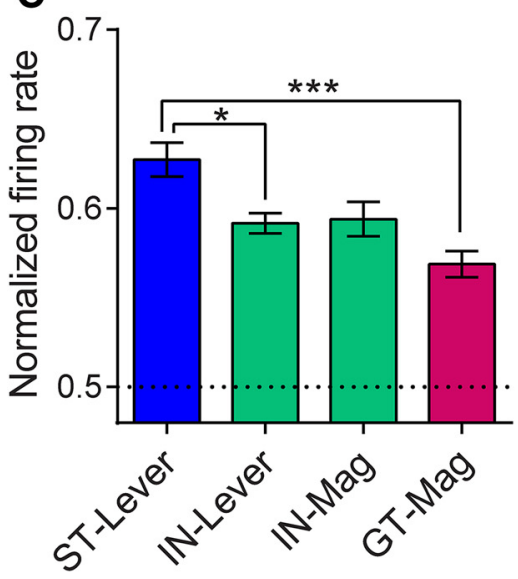

D Lever interaction

$\triangle$ Food cup interaction

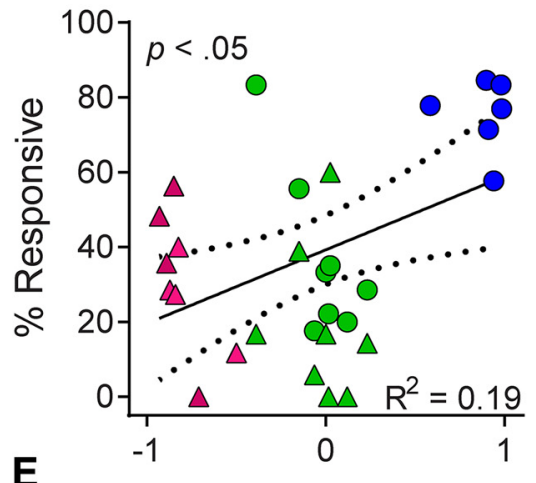

E

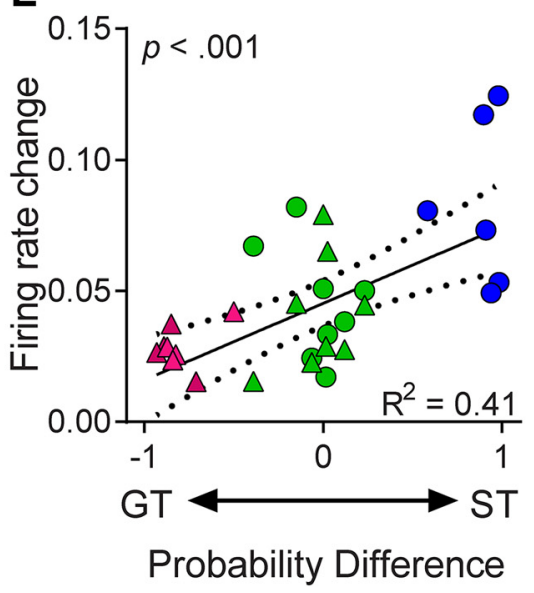

Figure 8. Although the $7 \mathrm{~s}$ cue period described above captured periods of sign- and goal-tracking, there was some variation in behavior from trial to trial. Therefore, we isolated the portion of each trial in which rats were actively engaged with the lever or magazine. $\boldsymbol{A}$, Example ratings of lever and magazine interaction intervals for individual trials. $\boldsymbol{B}$, STs had significantly more cells that were responsive during their interaction with the lever CS than INs during interactions with either the lever $C S$ or the food magazine and than GTs during interactions with the food magazine; ${ }^{* * *} p<0.001$. This was true for excitatory and inhibitory responses, as STs had more of both than INs and GTs; $\# p<0.05-0.001$. C, The increase in firing during interaction intervals (among excitatory cells only) was significantly greater in STs than GTs and IN-Lever; ${ }^{*} p<0.05$, ${ }^{* *} p<0.001$. There were significant correlations between probability difference scores and the percentage of responsive cells $(\boldsymbol{D})$ and the firing rate change for individual rats $(\boldsymbol{E})$.

(Flagel et al., 2009; Saunders and Robinson, 2013; Robinson et al., 2014). If neural activity in the VP encoded only the predictive value of the cue and thus its ability to evoke a CR, then there should be no group differences in neural activity during the CS period. However, there were pronounced group differences during the CS period, but not during the period after CS offset, when the food pellet was retrieved and consumed. This suggests that a major component of the information transmitted by the VP is related to variation in the degree to which the CS acquired incentive stimulus properties. Conversely, all rats (except those in the unpaired group) did show cue-induced changes in VP firing (al-
Lever interaction

Food cup interaction

- Lever press

$\Delta$ Food cup entry beit less than in STs), suggesting that the lever CS may not be completely devoid of incentive properties in INs and GTs and/or that the VP encodes a mixture of predictive and incentive signals.

The moment of CS onset is the unexpected first predictor of all upcoming task events, including the reward, and previous studies have reported that the immediate VP response to cue onset reflects a predictive signal that is unaffected by changes in the strength of incentive motivation (Tindell et al., 2005; Zhang et al., 2009; Smith et al., 2011). The fact that we saw group differences in response to CS onset is not consistent with the idea that this represents a purely predictive signal. Group differences during CS onset were weaker than those seen later in the trial, however, suggesting that this phasic response may be more closely related to reward prediction than the sustained cue responses that follow it. It is also worth noting that all rats (STs, GTs, and INs) developed a conditioned orienting response to the lever CS, which occurred before they approached the lever or the food magazine and it is difficult to determine the extent to which this contributed to similarities in the CS onset effect.

The pattern of responses to task events was variable, but two features stand out. In STs, the most common pattern was an increase in firing that began with CS onset and was sustained throughout the rest of the trial (also see Howe et al., 2013; Collins et al., 2016), even outlasting reward delivery and consumption. The second activation pattern had few sustained responses and this best characterized GTs. This pattern consisted of phasic bursts of activity changes to discrete task events (CS onset, CS offset, and US consumption). This second pattern resembles the VP firing observed in previous studies in which a second cue response (equivalent to CS offset) was identified as being responsible for the incentive signal (for details, see Tindell et al., 2005; Zhang et al., 2009; Smith et al., 2011). One very important difference between these studies is that the earlier studies used a tone CS. The CRs evoked by tones are different from those evoked by discrete light or lever cues (Holland, 1977). Importantly, a tone CS does not support a ST CR in rats and a tone CS is a less effective incentive stimulus than a lever CS (Holland et al., 2014; Meyer et al., 2014; Beckmann and Chow, 2015). Therefore, variation in neural activity related to the motivational properties of a cue may be more readily apparent using a lever CS than a tone CS.

INs

INs alternated CRs directed toward the lever and magazine, often interacting with both during a single trial, and it might be ex- 


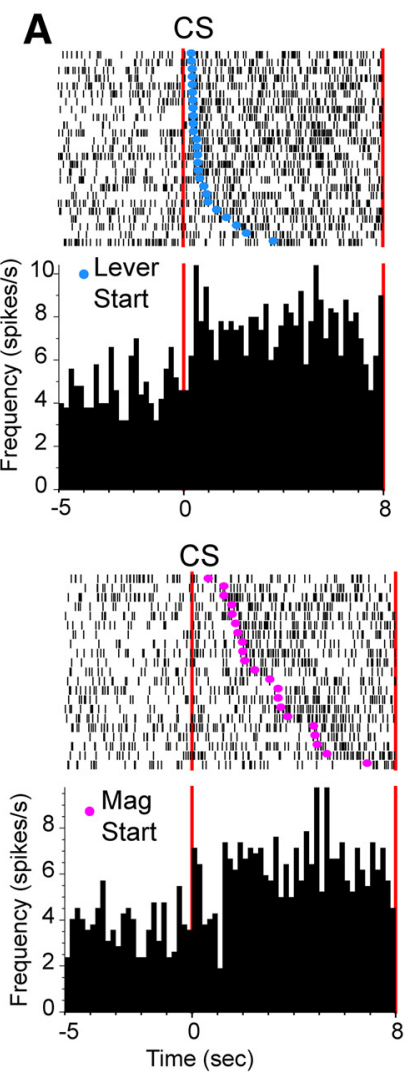

E

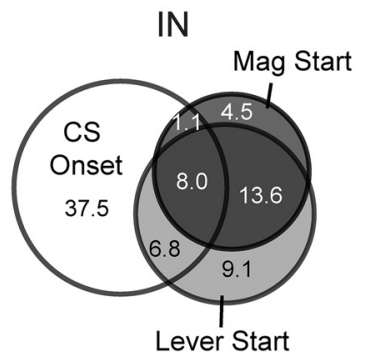

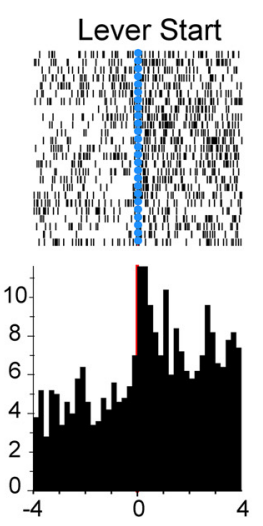

Mag Start
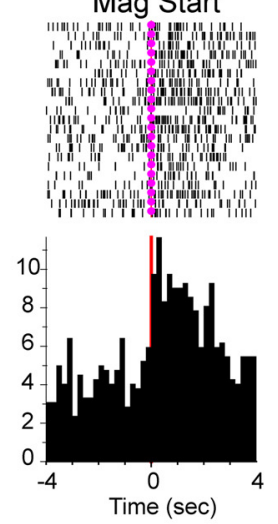

$\mathbf{F}$

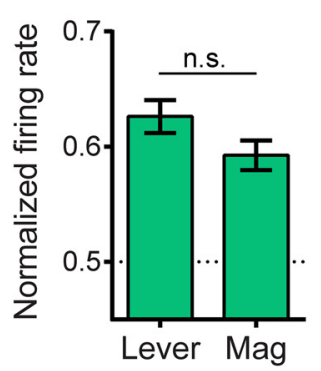

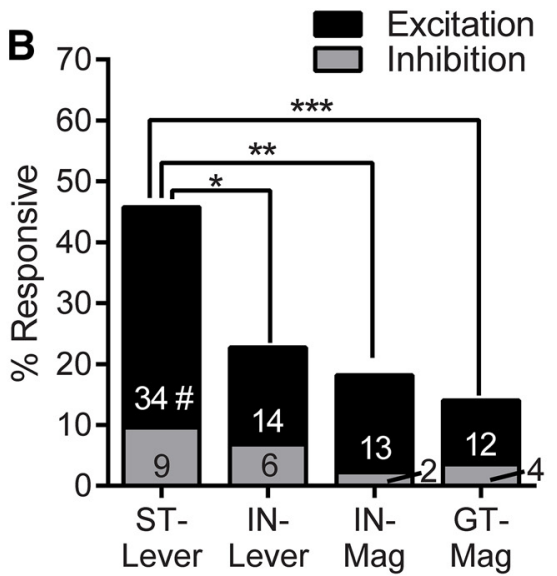
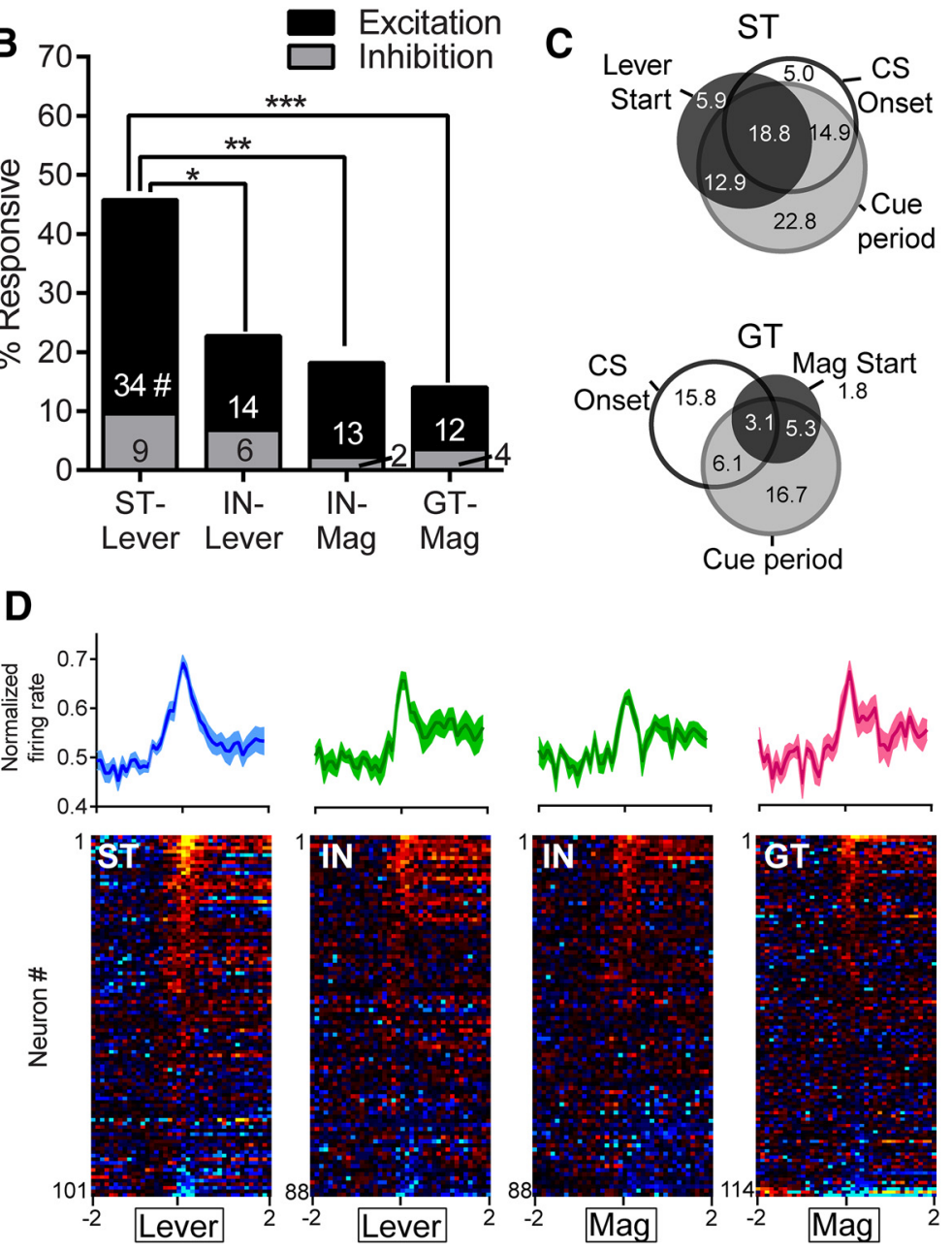

$0.2 \quad 0.4 \quad 0.6 \quad 0.8$

Excitation

Figure 9. VP neural activity at the moment of initial contact with the lever or magazine. $A$, Example rasters and histograms are shown for a single ST cell (top) and a single GT cell (bottom) that were responsive both to task events and the start of lever or magazine interaction ("lever start" and "mag start," respectively). Left panels show spikes aligned to CS onset, with trials sorted by the latency of lever start or mag start. In the right panels, the same cells show clear excitatory responses when aligned to lever start or mag start. $\boldsymbol{B}$, STs had significantly more cells responsive to Lever Start than GTs and INs had responsive to either Lever Start or Mag Start; ${ }^{*} p<0.05,{ }^{* * *} p<0.001$. These group differences were observed among excitatory responses, but not inhibitory responses; \#STs significantly greater than IN-Lever $(p<0.05)$, IN-Mag $(p<0.001)$, and GT-Mag ( $p<0.001)$. C, Venn diagrams showing that the majority of contact-responsive neurons in STs and GTs were also considered responsive to CS onset and/or the cue period (numbers are percentage responsive to each combination). D, Heat maps showing firing aligned to initial lever or magazine contact. Figures above show the average magnitude of excitation in that group (100 ms bins). Although the population response was stronger in STs (in terms of percentage), the magnitude of responses did not differ between groups. $\boldsymbol{E}$, INs had cells that were responsive to both lever and magazine start, as well as other task events. Venn diagrams show the overlap of responses to lever start, mag start, and CS onset. F, Among IN cells that showed excitatory responses to both lever and mag start, there was no significant difference in the magnitude of responses.

pected that neural activity would vary depending on the CR emitted. For example, dopamine blockade reduces lever-directed behavior, but not magazine-directed behavior, in INs (Saunders and Robinson, 2012). However, we found no such difference in the profile of neural activation. Perhaps attraction to the lever needs to be strong enough to dominate behavior before it is reflected in VP firing. The fact that the neural activity of INs did not vary based on behavior addresses one potential concern that is inherent to the design of this experiment: that neural activity during the interaction period necessarily coincides with motor actions that differ to some extent between STs and GTs. Although ST and GT CRs share many of the same action patterns (DiFeliceantonio and Berridge, 2012), there are also differences, which raises the possibility that enhanced firing in STs may have been influenced by the specific movements that are more prominent in ST than in GT (such as biting and pressing on the lever). If greater cell firing during lever interaction were due to particular movement patterns, then one would expect INs to show greater firing during periods of lever interaction than magazine interaction. However, this was not the case, which supports the idea that enhanced activation in STs represents an internal motivational state (Movie 1).

\section{Reward-related responses}

It has been shown previously that VP neurons respond to food reward even after CS-US associations are well learned, with the 
strength of neural changes reflecting the intensity of hedonic responses (Tindell et al., 2006; Smith et al., 2011). Consistent with this, VP neurons of all animals showed prominent activation related to reward delivery and consumption, although we do not have any measures of hedonic responses and cannot address that issue directly. However, if the food had a different value in STs and GTs, then they should differ in the acquisition of conditioned responding, but they do not.

\section{Neural circuitry}

Both core and shell subregions of the nucleus accumbens heavily innervate the VP, and the VP has been referred to as the "limbic final common pathway," where motivational signals get translated into actions (Mogenson and Yang, 1991; Smith et al., 2009; Ho and Berridge, 2013; Root et al., 2015). Although differences in dopamine activity in the core of STs relative to GTs (Flagel et al., 2011b) may have influenced the VP activity described here, the contribution of different inputs to the VP activity is unknown. Finally, the present study focused only on the posterior VP, but the VP is a heterogeneous structure with differences in cell morphology and connectivity patterns along the rostral-caudal axis (Kupchik and Kalivas, 2013) and the anterior and posterior regions of the VP often play different roles in reward-related behavior (Smith and Berridge, 2005, 2007; Root et al., 2010, 2013; Leung and Balleine, 2013; Mahler et al., 2014).

In conclusion, variation in neural activity in the VP was associated with variation in the degree to which a food cue was attributed with incentive salience, but not with the predictive value of the cue, consistent with previous studies on the neurobiology of STs and GTs (Danna and Elmer, 2010; Flagel et al., 2011a, 2011b; Clark et al., 2012; Danna et al., 2013; Haight et al., 2015; Singer et al., 2016; Yager et al., 2015). We suggest, therefore, that the VP may play an especially important role in the neural processes by which reward-paired cues bias attention, attract individuals into close proximity to them, and motivate seeking behavior, which, under some circumstances, can lead to maladaptive behavior, including overeating and addiction.

\section{References}

Ambroggi F, Ghazizadeh A, Nicola SM, Fields HL (2011) Roles of nucleus accumbens core and shell in incentive-cue responding and behavioral inhibition. J Neurosci 31:6820-6830. CrossRef Medline

Avila I, Lin SC (2014a) Motivational salience signal in the basal forebrain is coupled with faster and more precise decision speed. PLoS Biol 12: e1001811. CrossRef Medline

Avila I, Lin SC (2014b) Distinct neuronal populations in the basal forebrain encode motivational salience and movement. Front Behav Neurosci 8:421. CrossRef Medline

Beckmann JS, Chow JJ (2015) Isolating the incentive salience of rewardassociated stimuli: value, choice, and persistence. Learn Mem 22: 116-127. CrossRef Medline

Cardinal RN, Parkinson JA, Hall J, Everitt BJ (2002) Emotion and motivation: the role of the amygdala, ventral striatum, and prefrontal cortex. Neurosci Biobehav Rev 26:321-352. CrossRef Medline

Castro DC, Berridge KC (2014) Advances in the neurobiological bases for food 'liking' versus 'wanting'. Physiol Behav 136:22-30. CrossRef Medline

Chang SE, Todd TP, Bucci DJ, Smith KS (2015) Chemogenetic manipulation of ventral pallidal neurons impairs acquisition of sign-tracking in rats. Eur J Neurosci 42:3105-3116. CrossRef Medline

Clark JJ, Hollon NG, Phillips PE (2012) Pavlovian valuation systems in learning and decision making. Curr Opin Neurobiol 22:1054-1061. CrossRef Medline

Collins AL, Greenfield VY, Bye JK, Linker KE, Wang AS, Wassum KM (2016) Dynamic mesolimbic dopamine signaling during action sequence learning and expectation violation. Sci Rep 6:20231. CrossRef Medline

Cromwell HC, Berridge KC (1993) Where does damage lead to enhanced food aversion: the ventral pallidum substantia innominata or lateral hypothalamus. Brain Res 624(1-2):1-10.

Danna CL, Elmer GI (2010) Disruption of conditioned reward association by typical and atypical antipsychotics. Pharmacol Biochem Behav 96: 40-47. CrossRef Medline

Danna CL, Shepard PD, Elmer GI (2013) The habenula governs the attribution of incentive salience to reward predictive cues. Front Hum Neurosci 7:781. CrossRef Medline

Day JJ, Wheeler RA, Roitman MF, Carelli RM (2006) Nucleus accumbens neurons encode Pavlovian approach behaviors: evidence from an autoshaping paradigm. Eur J Neurosci 23:1341-1351. CrossRef Medline

DiFeliceantonio AG, Berridge KC (2012) Which cue to 'want'? Opioid stimulation of central amygdala makes goal-trackers show stronger goaltracking, just as sign-trackers show stronger sign-tracking. Behav Brain Res 230:399-408. CrossRef Medline

Flagel SB, Watson SJ, Robinson TE, Akil H (2007) Individual differences in the propensity to approach signals vs goals promote different adaptations in the dopamine system of rats. Psychopharmacology (Berl) 191:599-607. CrossRef Medline

Flagel SB, Akil H, Robinson TE (2009) Individual differences in the attribution of incentive salience to reward-related cues: implications for addiction. Neuropharmacology 56:139-148. CrossRef Medline

Flagel SB, Cameron CM, Pickup KN, Watson SJ, Akil H, Robinson TE (2011a) A food predictive cue must be attributed with incentive salience for it to induce c-fos mRNA expression in cortico-striatal-thalamic brain regions. Neuroscience 196:80-96. CrossRef Medline

Flagel SB, Clark JJ, Robinson TE, Mayo L, Czuj A, Willuhn I, Akers CA, Clinton SM, Phillips PE, Akil H (2011b) A selective role for dopamine in stimulus-reward learning. Nature 469:53-57. CrossRef Medline

Haight JL, Fraser KM, Akil H, Flagel SB (2015) Lesions of the paraventricular nucleus of the thalamus differentially affect sign- and goal-tracking conditioned responses. Eur J Neurosci 42:2478-2488. CrossRef Medline

Ho CY, Berridge KC (2013) An orexin hotspot in ventral pallidum amplifies hedonic 'liking' for sweetness. Neuropsychopharmacology 38: 1655-1664. CrossRef Medline

Holland PC (1977) Conditioned stimulus as a determinant of the form of the Pavlovian conditioned response. J Exp Psychol Anim Behav Process 3:77-104. CrossRef Medline

Holland PC, Asem JS, Galvin CP, Keeney CH, Hsu M, Miller A, Zhou V (2014) Blocking in autoshaped lever-pressing procedures with rats. Learn Behav 42:1-21. CrossRef Medline

Howe MW, Tierney PL, Sandberg SG, Phillips PE, Graybiel AM (2013) Prolonged dopamine signalling in striatum signals proximity and value of distant rewards. Nature 500:575-579. CrossRef Medline

Kupchik YM, Kalivas PW (2013) The rostral subcommissural ventral pallidum is a mix of ventral pallidal neurons and neurons from adjacent areas: an electrophysiological study. Brain Struct Funct 218:1487-1500. CrossRef Medline

Leung BK, Balleine BW (2013) The ventral striato-pallidal pathway mediates the effect of predictive learning on choice between goal-directed actions. J Neurosci 33:13848-13860. CrossRef Medline

Mahler SV, Berridge KC (2009) Which cue to "want?" Central amygdala opioid activation enhances and focuses incentive salience on a prepotent reward cue. J Neurosci 29:6500-6513. CrossRef Medline

Mahler SV, Vazey EM, Beckley JT, Keistler CR, McGlinchey EM, Kaufling J, Wilson SP, Deisseroth K, Woodward JJ, Aston-Jones G (2014) Designer receptors show role for ventral pallidum input to ventral tegmental area in cocaine seeking. Nat Neurosci 17:577-585. CrossRef Medline

McGinty VB, Lardeux S, Taha SA, Kim JJ, Nicola SM (2013) Invigoration of reward seeking by cue and proximity encoding in the nucleus accumbens. Neuron 78:910-922. CrossRef Medline

Meyer PJ, Lovic V, Saunders BT, Yager LM, Flagel SB, Morrow JD, Robinson TE (2012) Quantifying individual variation in the propensity to attribute incentive salience to reward cues. PLoS One 7:e38987. CrossRef Medline

Meyer PJ, Cogan ES, Robinson TE (2014) The form of a conditioned stimulus can influence the degree to which it acquires incentive motivational properties. PLoS One 9:e98163. CrossRef Medline

Mogenson GJ, Yang CR (1991) The contribution of basal forebrain to limbic-motor integration and the mediation of motivation to action. Adv Exp Med Biol 295:267-290. CrossRef Medline

Paxinos G, Watson C (2007) The rat brain in stereotaxic coordinates. New York: Academic. 
Robinson TE, Berridge KC (2008) Review. The incentive sensitization theory of addiction: some current issues. Philos Trans R Soc Lond B Biol Sci 363:3137-3146. CrossRef Medline

Robinson TE, Flagel SB (2009) Dissociating the predictive and incentive motivational properties of reward-related cues through the study of individual differences. Biol Psychiatry 65:869-873. CrossRef Medline

Robinson TE, Yager LM, Cogan ES, Saunders BT (2014) On the motivational properties of reward cues: Individual differences. Neuropharmacology 76:450-459. CrossRef Medline

Root DH, Fabbricatore AT, Ma S, Barker DJ, West MO (2010) Rapid phasic activity of ventral pallidal neurons during cocaine self-administration. Synapse 64:704-713. CrossRef Medline

Root DH, Ma S, Barker DJ, Megehee L, Striano BM, Ralston CM, Fabbricatore AT, West MO (2013) Differential roles of ventral pallidum subregions during cocaine self-administration behaviors. J Comp Neurol 521: 558-588. CrossRef Medline

Root DH, Melendez RI, Zaborszky L, Napier TC (2015) The ventral pallidum: Subregion-specific functional anatomy and roles in motivated behaviors. Prog Neurobiol 130:29-70. CrossRef Medline

Saunders BT, Robinson TE (2010) A cocaine cue acts as an incentive stimulus in some but not others: implications for addiction. Biol Psychiatry 67:730-736. CrossRef Medline

Saunders BT, Robinson TE (2012) The role of dopamine in the accumbens core in the expression of Pavlovian-conditioned responses. Eur J Neurosci 36:2521-2532. CrossRef Medline

Saunders BT, Robinson TE (2013) Individual variation in resisting temptation: implications for addiction. Neurosci Biobehav Rev 37:1955-1975. CrossRef Medline

Saunders BT, Yager LM, Robinson TE (2013) Cue-evoked cocaine "craving": role of dopamine in the accumbens core. J Neurosci 33:1398914000. CrossRef Medline

Schultz W, Dayan P, Montague PR (1997) A neural substrate of prediction and reward. Science 275:1593-1599. CrossRef Medline

Shimura T, Imaoka H, Yamamoto T (2006) Neurochemical modulation of ingestive behavior in the ventral pallidum. Eur J Neurosci 23:1596-1604. CrossRef Medline

Singer BF, Guptaroy B, Austin CJ, Wohl I, Lovic V, Seiler JL, Vaughan RA, Gnegy ME, Robinson TE, Aragona BJ (2016) Individual variation in incentive salience attribution and accumbens dopamine transporter expression and function. Eur J Neurosci 43:662-670. CrossRef Medline

Smith KS, Berridge KC (2005) The ventral pallidum and hedonic reward: neurochemical maps of sucrose "liking" and food intake. J Neurosci 25: 8637-8649. CrossRef Medline

Smith KS, Berridge KC (2007) Opioid limbic circuit for reward: interaction between hedonic hotspots of nucleus accumbens and ventral pallidum. J Neurosci 27:1594-1605. CrossRef Medline
Smith KS, Tindell AJ, Aldridge JW, Berridge KC (2009) Ventral pallidum roles in reward and motivation. Behav Brain Res 196:155-167. CrossRef Medline

Smith KS, Berridge KC, Aldridge JW (2011) Disentangling pleasure from incentive salience and learning signals in brain reward circuitry. Proc Nat Acad Sci U S A 108:E255-E264. CrossRef Medline

Stefanik MT, Kupchik YM, Brown RM, Kalivas PW (2013) Optogenetic evidence that pallidal projections, not nigral projections, from the nucleus accumbens core are necessary for reinstating cocaine seeking. J Neurosci 33:13654-13662. CrossRef Medline

Tachibana Y, Hikosaka O (2012) The primate ventral pallidum encodes expected reward value and regulates motor action. Neuron 76:826-837. CrossRef Medline

Tindell AJ, Berridge KC, Zhang J, Peciña S, Aldridge JW (2005) Ventral pallidal neurons code incentive motivation: amplification by mesolimbic sensitization and amphetamine. Eur J Neurosci 22:2617-2634. CrossRef Medline

Tindell AJ, Smith KS, Peciña S, Berridge KC, Aldridge JW (2006) Ventral pallidum firing codes hedonic reward: when a bad taste turns good. J Neurophysiol 96:2399-2409. CrossRef Medline

Tindell AJ, Smith KS, Berridge KC, Aldridge JW (2009) Dynamic computation of incentive salience: "wanting" what was never "liked." J Neurosci 29:12220-12228. CrossRef Medline

Tomie A, Aguado AS, Pohorecky LA, Benjamin D (2000) Individual differences in Pavlovian autoshaping of lever pressing in rats predict stressinduced corticosterone release and mesolimbic levels of monoamines. Pharmacol Biochem Behav 65:509-517. CrossRef Medline

Tomie A, Grimes KL, Pohorecky LA (2008) Behavioral characteristics and neurobiological substrates shared by Pavlovian sign-tracking and drug abuse. Brain Res Rev 58:121-135. CrossRef Medline

Waelti P, Dickinson A, Schultz W (2001) Dopamine responses comply with basic assumptions of formal learning theory. Nature 412:43-48. CrossRef Medline

Wan X, Peoples LL (2006) Firing patterns of accumbal neurons during a Pavlovian-conditioned approach task. J Neurophysiol 96:652-660. CrossRef Medline

Yager LM, Robinson TE (2013) A classically conditioned cocaine cue acquires greater control over motivated behavior in rats prone to attribute incentive salience to a food cue. Psychopharmacology (Berl) 226: 217-228. CrossRef Medline

Yager LM, Pitchers KK, Flagel SB, Robinson TE (2015) Individual variation in the motivational and neurobiological effects of an opioid cue. Neuropsychopharmacology 40:1269-1277. CrossRef Medline

Zhang J, Berridge KC, Tindell AJ, Smith KS, Aldridge JW (2009) A neural computational model of incentive salience. PLoS Comput Biol 5:e1000437. CrossRef Medline 